\title{
WHITNEY'S EXTENSION THEOREM IN O-MINIMAL STRUCTURES
}

\author{
ATHIPAT THAMRONGTHANYALAK
}

\begin{abstract}
In 1934, Whitney gave a necessary and sufficient condition on a jet of order $m$ on a closed subset $E$ of $\mathbb{R}^{n}$ to be the jet of order $m$ of a $C^{m}$-function; jets satisfying this condition are known as $C^{m}$-Whitney fields. Later, Pawłucki and Kurdyka proved that subanalytic $C^{m}$-Whitney fields are jets of order $m$ of sybanalytic $C^{m}$-functions. Here, we work in an o-minimal expansion of a real closed field and prove a definable version of Whitney's Extension Theorem: every definable $C^{m}$-Whitney field is a jet of order $m$ of a definable $C^{m}$-function.
\end{abstract}

\section{INTRODUCTION}

Whitney's Extension Theorem, which can be considered as a partial converse of Taylor's Theorem, was proved by H. Whitney in 1934. (See $[7,10]$ for the proof, and $[11,12]$ for related problems.) It roughly says that if $f: E \rightarrow \mathbb{R}$, where $E$ is a closed subset of $\mathbb{R}^{n}$, can be approximated by Taylor polynomials of degree $m$ in a certain uniform way (as entailed by Taylor's Formula), then $f$ can be extended to a $C^{m}$ function on $\mathbb{R}^{n}$. A $C^{m}$-Whitney field on $E$ encodes the data relevant for such an approximation of $f$. The present paper is motivated by the work of K. Kurdyka and W. Pawłucki [6], who proved a version of Whitney's Extension Theorem in the category of subanalytic functions.

Throughout this paper, let $R$ be a real closed ordered field and $\boldsymbol{R}$ be an o-minimal expansion of $R$ in a language $\mathscr{L}$. Moreover, "definable" always means "definable in $\boldsymbol{R}$, possibly with parameters." As usual, a map is called definable if its graph is. In the main bulk of the paper, we assume that the reader is familiar with the basic definitions and facts concerning o-minimal structures; see, e.g., $[1,2]$. For the purposes of this introduction, the reader can think of the special case where $R$ is the usual ordered field of real numbers $\mathbb{R}$, so the sets definable in $\boldsymbol{R}$ are precisely the semialgebraic sets (i.e., defined by finite boolean combinations of polynomial inequalities). In this paper, we prove Whitney's Extension Theorem for definable $C^{m}$-Whitney fields.

Theorem (Whitney's Extension Theorem). Let $F$ be a definable $C^{m}$-Whitney field on a closed subset $E$ of $R^{n}$. Then for each $q \geq m, F$ has a definable $C^{m}$-extension which is $C^{q}$ on $R^{n} \backslash E$.

Let us make precise what we mean by a definable $C^{m}$-Whitney field and an extension of such a Whitney field. Let $E \subseteq R^{n}$ be definable. A (definable) jet of order $m$ on $E$ is a family $F=\left(F^{\alpha}\right)_{|\alpha| \leq m}$ where each $F^{\alpha}: E \rightarrow R$ is a definable

Date: September 4, 2013. 
continuous function. If $F$ is a jet of order $m$ on $E$ and $E^{\prime} \subseteq E$ is definable, then $F\left\lceil E^{\prime}:=\left(F^{\alpha} \mid E^{\prime}\right)_{|\alpha| \leq m}\right.$ is a jet of order $m$ on $E^{\prime}$. If $E$ is open, then for each definable $C^{m}$-function $f: E \rightarrow R$, we obtain a jet $J^{m}(f)=\left(D^{\alpha} f\right)_{|\alpha| \leq m}$ of order $m$ on $E$. Here, $\alpha=\left(\alpha_{1}, \ldots, \alpha_{n}\right)$ ranges over $\mathbb{N}^{n}$, and we let $D^{\alpha}=\frac{\partial^{\alpha_{1}}}{\partial x_{1}^{\alpha_{1}}} \cdots \frac{\partial^{\alpha_{n}}}{\partial x_{n}^{\alpha_{n}}}$ and $|\alpha|:=\alpha_{1}+\cdots+\alpha_{n}$. Now for every $x \in R^{n}, a \in E$, and $F$ a jet of order $m$ on $E$, set

$$
\begin{aligned}
& T_{a}^{m} F(x)=\sum_{|\alpha| \leq m} F^{\alpha}(a) \frac{(x-a)^{\alpha}}{\alpha !}, \\
& R_{a}^{m} F(x)=F-J^{m}\left(T_{a}^{m} F(x)\right) .
\end{aligned}
$$

We say that a jet $F$ of order $m$ is a definable $C^{m}$-Whitney field on $E\left(F \in \mathscr{E}^{m}(E)\right)$ if, for all $x_{0} \in E$ and $|\alpha| \leq m$, we have

$$
\left(R_{x}^{m} F\right)^{\alpha}(y)=o\left(|x-y|^{m-|\alpha|}\right) \quad \text { as } E \ni x, y \rightarrow x_{0} ;
$$

equivalently, if for all for $x_{0} \in E$ and $z \in R^{n}$,

$$
\left|T_{x}^{m} F(z)-T_{y}^{m} F(z)\right|=o\left(|x-z|^{m}+|y-z|^{m}\right) \quad \text { as } E \ni x, y \rightarrow x_{0} .
$$

(See [7].) Note that if $F \in \mathscr{E}^{m}(E)$ and $E^{\prime} \subseteq E$ is definable, then $F\left\lceil E^{\prime} \in \mathscr{E}^{m}\left(E^{\prime}\right)\right.$. Also, if $E$ is open and $f: E \rightarrow R$ is a definable $C^{m}$-function, then $J^{m}(f)$ is a $C^{m_{-}}$ Whitney field, by Taylor's Theorem. Given $F \in \mathscr{E}^{m}(E)$, we say that a definable $C^{m}$-function $f: R^{n} \rightarrow R$ is an extension of $F$ if $J^{m}(f)\lceil E=F$.

An immediate consequence of the theorem above is the following:

Corollary. Suppose that $E$ is regular closed (i.e., E equals the closure of its interior). Let $f: E \rightarrow R$ be a definable function such that for each $x \in E$ there is an open neighborhood $U$ of $x$ in $R^{n}$ and an extension of $f \uparrow(E \cap U)$ to a definable $C^{m}$-function $U \rightarrow R$. Then $f$ extends to a definable $C^{m}$-function $R^{n} \rightarrow R$.

Key ingredients in the construction of Kurdyka and Pawłucki are partitions of unity and 1-regularity, which are not generally available in o-minimal expansions of real closed fields. In the case where $R=\mathbb{R}$ is the usual ordered field of reals, their method only applies in the situation where $E$ is compact. In [9], Pawtucki introduced a new algorithm to extend $C^{m}$-Whitney fields on $E \subseteq \mathbb{R}^{n}$. However, this new construction doesn't preserve definability in a given o-minimal expansion of $\mathbb{R}$, due to its use of integration. (Pawłucki's extension operator, on the other hand, has the virtue of being linear.) In this paper we still follow Pawłucki's five-step strategy for extending $C^{m}$-Whitney fields from [9], while combining it with Fischer's $\Lambda^{m}$ regular Stratification Theorem from [3]. This theorem allows us to prove analogues of Pawłucki's main lemmas (such as his " $\Lambda^{m}$-regular Decomposition Theorem") in our given o-minimal structure $\boldsymbol{R}$.

Organization of the paper. In the first section, we focus on geometry of definable sets. First, we show some basic facts which will be repeatedly used throughout this paper. Then important terminology, such as $\Lambda^{m}$-regularity and $\epsilon$-flatness, will be introduced. Once all necessary terminology is ready, we will state the main result 
of [3], which is an important tool in later proofs. Also, several modifications of lemmas in [3] will be provided in this section.

In Sections 2 and 3, we study the separation property of a pair of subset of $R^{n}$ and prove the $\Lambda^{m}$-regular Separation Theorem, which is a purely o-minimal version of the $\Lambda^{m}$-regular Decomposition Theorem from [8]. The proof of the $\Lambda^{m}$-regular Decomposition Theorem in that paper involves concepts which are not valid in a general o-minimal context, for example, the length of rectificable curves. In our situation, these concepts will be replaced by control over differences between tangent spaces ( $\epsilon$-flatness). In Section 3, the notion of $\Lambda^{m}$-pancake, which is central for the statement of $\Lambda^{m}$-regular Separation Theorem, will be given, followed by the full statement of this theorem and its proof.

In Section 4, we study properties of $C^{m}$-Whitney fields and related concepts. The main results of this section are special cases of Whitney's Extension Theorem. Let $\Omega \subseteq R^{d}$ be open and definable. We work with a definable $C^{m}$-Whitney field $F:=$ $\left(F^{\alpha}\right)_{|\alpha| \leq m}$ on $\Omega \times\{0\}^{l}$, and we show that if each function $F^{\alpha}: \Omega \rightarrow R$ is $\Lambda^{m}$-regular as defined in Section 1, then $F$ has a definable $C^{m}$-extension.

In the last two sections, we follow Pawłucki's five-step strategy from [9]. However, as we mentioned earlier, Pawłucki's construction doesn't preserves definability. Integration and 1-regularity are key in his construction and they are not generally available in a fixed general o-minimal structure. These two concepts will be replaced by Fischer's $\Lambda^{m}$-regular Stratification Theorem, the special cases established in Section 4 , and pervasive use of $\epsilon$-flatness.

Conventions and notations. Throughout this paper, $d, k, m, n$, and $q$ will range over the set $\mathbb{N}=\{0,1,2,3, \ldots\}$ of natural numbers. Given a map $f: X \rightarrow Y$ we write

$$
\Gamma(f)=\{(x, f(x)): x \in X\} \subseteq X \times Y
$$

for the graph of $f$. Let

$$
\begin{aligned}
C(X) & :=\{f: X \rightarrow R: f \text { is continuous and definable }\}, \\
C_{\infty}(X) & :=C(X) \cup\{+\infty,-\infty\},
\end{aligned}
$$

where $+\infty$ and $-\infty$ are considered as constant functions on $X$. For $f, g \in C_{\infty}(X)$ we write $f<g$ if $f(x)<g(x)$ for all $x \in X$, and in this case we set

$$
(f, g):=\{(x, r) \in X \times R: f(x)<r<g(x)\} .
$$

Similarly an interval in $R$ is a set of the form

$$
(a, b):=\{r \in R: a<r<b\} \quad \text { where } a, b \in R_{ \pm \infty}=R \cup\{-\infty,+\infty\} \text { and } a<b .
$$

For a set $S \subseteq R^{n}$ we denote by $\operatorname{cl} S=\operatorname{cl}(S)$ the closure, by $\partial S=\partial(S):=\operatorname{cl}(S) \backslash S$ the frontier, and by int $S=\operatorname{int}(S)$ the interior of $S$. We denote the Euclidean norm on $R^{n}$ by $\|\cdot\|$ and the associated metric by $(x, y) \mapsto d(x, y):=\|x-y\|$. For $X \subseteq R^{n}$, we say that $f: X \rightarrow R^{m}$ is Lipschitz if there is a rational $L>0$ such that

$$
\|f(x)-f(y)\| \leq L\|x-y\| \quad \text { for all } x, y \in X .
$$


Given $x \in R^{n}$, for a non-empty definable set $S \subseteq R^{n}$ let $d(x, S):=\inf _{y \in S} d(x, y) \in$ $R^{\geq 0}$ be the distance between $x$ and $S$, and $d(x, \emptyset):=+\infty$. Given a collection $\mathscr{C}$ of subsets of $R^{n}$, we let $\mathscr{C}^{o}:=\{C \in \mathscr{C}: C$ is open $\}$.

Acknowledgements. The results in this paper are part of the author's PhD thesis at UCLA, written under the guidance of Matthias Aschenbrenner. We would like to thank W. Pawłucki for an email exchange clarifying some arguments in [6]. The author acknowledges support by a Queen Sirikit Scholarship.

\section{Preliminaries}

In this section we introduce notations, terminologies, and basic facts which will be used repeatedly throughout this paper. Our notations mainly follow [3].

Definition 1.1. Let $X \subseteq E$ be definable subsets of $R^{n}$.

(1) We say that $X$ is a small subset of $E$ if $\operatorname{dim}(X)<\operatorname{dim}(E)$. We will often just write " $X$ is small" if the ambient set $E$ is clear from the context.

(2) We say that $X$ is a large subset of $E(X \Subset E)$ if $E \backslash X$ is small.

Note that if the definable set $X$ is a large subset of the definable set $E$, and $Y$ is any definable set of $\operatorname{dimension} \operatorname{dim}(Y)=\operatorname{dim}(E)$, then $X \cap Y$ is non-empty (and, actually, a large subset of $Y$ ).

Definition 1.2. Let $E$ be a definable subset of $R^{n}$. For each $x \in E$, we say that $E$ is of local dimension $d\left(\operatorname{dim}_{x}(E)=d\right)$ if there exists a definable open neighborhood $V$ of $X$ in $R^{n}$ such that $\operatorname{dim}(E \cap U)=d$ for every definable open neighborhood $U$ of $x$ in $V$. Moreover, we say that $E$ is of constant local dimension $d$ if $\operatorname{dim}_{x}(E)=d$ for every $x \in E$, equivalently, for every $x \in E$ and every definable open neighborhood $V$ of $x$ in $R^{n}, \operatorname{dim}(E \cap V)=d$. (Note that then $\operatorname{dim}(E)=d$.) We also say that $E$ is of constant local dimension if $E$ is of constant local dimension $d$ for some $d \in \mathbb{N}$.

The next proposition gives a condition ensuring that the closure of the graph of a continuous definable map may be recovered from its restriction to a large set. This fact will be useful in reducing later proofs to simpler cases.

Proposition 1.3. Let $\varphi: \Omega \rightarrow R^{n}$ be a continuous definable map, where $\Omega$ is a nonempty definable subset of $R^{k}$ of constant local dimension, and let $U \Subset \Omega$ be definable. Then $\operatorname{cl} \Gamma(\varphi)=\operatorname{cl} \Gamma(\varphi \mid U)$.

Proof. Let $E:=\operatorname{cl} \Gamma(\varphi)$. It is enough to show that $E \subseteq \operatorname{cl} \Gamma(\varphi \mid U)$. Let $x \in E$. Let $V$ be a definable open neighborhood of $x$. Then $V \cap \Gamma(\varphi) \neq \emptyset$. Note that $\pi(V \cap$ $\Gamma(\varphi)$ ), where $\pi: R^{k+n} \rightarrow R^{k}$ is the natural projection onto the first $k$ coordinates, is of dimension $d$ (since $\Omega$ is of constant local dimension). Since $U \Subset \Omega$, the set $U \cap \pi(V \cap \Gamma(\varphi))$ is non-empty. In particular, $V \cap \Gamma(\varphi \mid U) \neq \emptyset$. Since $V$ is arbitrary, $x \in \operatorname{cl} \Gamma(\varphi \mid U)$.

Remark. Let $E=\bigcup_{i=1}^{N} \operatorname{cl} \Gamma\left(\varphi_{i}\right)$ where each $\Omega_{i}$ is an open definable subset of $R^{k}$ and $\varphi_{i}: \Omega_{i} \rightarrow R^{n}$ is continuous and definable. Then by Proposition 1.3, we can replace 
the $\varphi_{i}$ by suitable restrictions to reduce to the case that for all $i, j$ with $\Omega_{i} \cap \Omega_{j} \neq \emptyset$ we have $\Omega_{i}=\Omega_{j}$.

One of our main tools is Fischer's theorem [3] on the existence of $\Lambda^{m}$-stratifications. We now state this theorem, and also prove some modifications of a few lemmas from [3]. For this, we need some definitions. In the following, we assume $m \geq 1$.

Definition 1.4. Let $f=\left(f_{1}, \ldots, f_{n}\right): \Omega \rightarrow R^{n}$ be a $C^{m}$-map, where $\Omega$ is a nonempty open subset of $R^{d}$, with $d \geq 1$. We say that $f$ is $\Lambda^{m}$-regular if there is some $L \in R^{>0}$ such that

$$
\left\|D^{\alpha} f(x)\right\| \leq \frac{L}{d(x, \partial \Omega)^{|\alpha|-1}} \quad \text { for all } x \in \Omega \text { and } \alpha \in \mathbb{N}^{d} \text { with } 1 \leq|\alpha| \leq m .
$$

Here and in the rest of the paper, for $\alpha=\left(\alpha_{1}, \ldots, \alpha_{d}\right) \in \mathbb{N}^{d}$ we set

$$
D^{\alpha}=\frac{\partial^{\alpha_{1}}}{\partial x_{1}^{\alpha_{1}}} \cdots \frac{\partial^{\alpha_{d}}}{\partial x_{d}^{\alpha_{d}}}, \quad|\alpha|:=\alpha_{1}+\cdots+\alpha_{d}
$$

and we let $D^{\alpha} f:=\left(D^{\alpha} f_{1}, \ldots, D^{\alpha} f_{n}\right)$ if $|\alpha| \leq m$ (so $\left.D^{0} f=f\right)$.

We also define every map $R^{0} \rightarrow R^{n}$ to be $\Lambda^{m}$-regular.

Notation. Let $\Omega \subseteq R^{d}$ be definable and open. Set

$$
\begin{aligned}
& \Lambda^{m}(\Omega):=\left\{f: \Omega \rightarrow R: f \text { is definable and } \Lambda^{m} \text {-regular }\right\}, \\
& \Lambda_{\infty}^{m}(\Omega):=\Lambda^{m}(\Omega) \cup\{-\infty,+\infty\},
\end{aligned}
$$

where $+\infty$ and $-\infty$ are considered as constant functions on $\Omega$.

Definition 1.5. Standard open $\Lambda^{m}$-regular cells in $R^{n}$ are defined inductively on $n$ as follows:

(1) $n=0: R^{0}$ is the standard open $\Lambda^{m}$-regular cell in $R^{0}$;

(2) $n \geq 1$ : a set of the form $(f, g)$ where $f, g \in \Lambda_{\infty}^{m}(D)$ such that $f<g$, and $D$ is a standard open $\Lambda^{m}$-regular cell in $R^{n-1}$.

We say that a subset of $R^{n}$ is a standard $\Lambda^{m}$-regular cell in $R^{n}$ if it is either a standard open $\Lambda^{m}$-regular cell in $R^{n}$ or one of the following:

(1) a singleton; or

(2) the graph of a definable $\Lambda^{m}$-regular map $D \rightarrow R^{n-d}$, where $D$ is a standard open $\Lambda^{m}$-regular cell in $R^{d}$, and $1 \leq d<n$.

A subset $E \subseteq R^{n}$ is called a $\Lambda^{m}$-regular cell in $R^{n}$ if there is a linear orthogonal transformation $\phi: R^{n} \rightarrow R^{n}$ such that $\phi(E)$ is a standard $\Lambda^{m}$-regular cell in $R^{n}$.

Even though in a general o-minimal expansion of a real closed field, we do not have a notion of angle (between two vectors) available, a substitute is provided by the terminology introduced in [3], which we recall next.

Definition 1.6. Let $R^{n \times n}$ be the space of all $n \times n$ matrices with entries from $R$, where $n \geq 1$, equipped with the operator norm $\|\cdot\|$. For each $d \leq n$, let

$$
\mathbb{H}_{n, d}=\left\{A \in R^{n \times n}: A^{\mathrm{t}}=A, A^{2}=A, \operatorname{tr}(A)=d\right\}
$$


be the subset of $R^{n \times n}$ consisting of the matrices (with respect to the standard basis of $R^{n}$ ) of orthogonal projections of $R^{n}$ onto a subspace of $R^{n}$, having trace $d$. Note that $\mathbb{H}_{n, d}$ is an algebraic subset of $R^{n \times n}$ (where $R^{n \times n}$ is identified with $R^{n^{2}}$ as usual) and hence definable. Consider

$$
\delta: \mathbb{H}_{n, d} \times \mathbb{H}_{n, d} \rightarrow R, \quad \delta(A, B)=\left\|B^{\perp} A\right\| \quad \text { where } B^{\perp}=\mathrm{id}-B,
$$

In [3] it is shown that $\delta$ is a metric on $\mathbb{H}_{n, d}$. For $A \in \mathbb{H}_{n, d}$ and $\epsilon>0$ let

$$
B_{\epsilon}(A)=\left\{B \in \mathbb{H}_{n, d}: \delta(B, A)<\epsilon\right\}
$$

be the open ball of radius $\epsilon$ centered at $A$ in $\mathbb{H}_{n, d}$, and let

$$
\overline{B_{\epsilon}}(A)=\left\{B \in \mathbb{H}_{n, d}: \delta(B, A) \leq \epsilon\right\}
$$

be the closed ball of radius $\epsilon$ centered at $A$ in $\mathbb{H}_{n, d}$. Fischer [3] also showed that for any rational $\epsilon>0, \mathbb{H}_{n, d}$ can be covered by finitely many balls of radius $\epsilon$.

In the rest of this section, $\epsilon$ ranges over rational numbers.

Definition 1.7. Let $M$ a definable $d$-dimensional (embedded) $C^{1}$-submanifold of $R^{n}$. We view the tangent bundle $T(M)$ of $M$ as a subbundle of $T\left(R^{n}\right) \cong R^{n} \times R^{n}$ in the natural way. Define $\tau_{M}: M \rightarrow \mathbb{H}_{n, d}$ by letting $\tau_{M}(x)$ be the matrix (w.r.t. the standard basis of $R^{n}$ ) of the orthogonal projection $R^{n} \rightarrow T_{x}(M)$. Let $A \in \mathbb{H}_{n, d}$ and $\epsilon>0$. We say that $M$ is $\epsilon$-flat with respect to $A$ if $\tau_{M}(M) \subseteq B_{\epsilon}(A)$.

A $d$-dimensional $C^{1}$-graph is a subset $M \subseteq R^{n}$ which, in a suitable coordinate system, is the graph of a definable $C^{1}$-map $\Omega \rightarrow R^{n-d}$, where $\Omega$ is a definable open subset of $R^{d}$. Note that every $d$-dimensional $C^{1}$-graph $M$ is a definable $d$-dimensional $C^{1}$-submanifold of $R^{n}$, hence the previous definition applies to $M$. Given $A \in \mathbb{H}_{n, d}$ and $\epsilon>0$, we also call a closed definable subset $E$ of $R^{n} \epsilon$-flat with respect to $A$ if $E$ is the closure of a finite disjoint union of $d$-dimensional $C^{1}$-graphs in $R^{n}$, each of which is $\epsilon$-flat with respect to $A$.

A standard $\Lambda^{m}$-regular cell of dimension $d$ is called $\epsilon$-flat if it is $\epsilon$-flat with respect to the projection of $R^{n}$ onto the first $d$ coordinates. In addition, we call a $\Lambda^{m}$-regular cell $\epsilon$-flat if there is a linear orthogonal transformation $\phi: R^{n} \rightarrow R^{n}$ such that the image of this set under $\phi$ is an $\epsilon$-flat standard $\Lambda^{m}$-regular cell.

Remark. Every $d$-dimensional $C^{1}$-cell in $R^{n}$ is a $d$-dimensional $C^{1}$-graph.

Proof. We will prove this remark by induction on $n$. For $n=0$, this remark is trivial. Suppose that, for every $d \leq n$, every $d$-dimensional $C^{1}$-cell in $R^{n}$ is a $d$-dimensional $C^{1}$-graph, and let $d \leq n+1$ and $C$ be a $d$-dimensional $C^{1}$-cell in $R^{n+1}$. If $C$ is an open cell, then $C$ is the graph of the map $C \rightarrow R^{0}$; it is obviously an $(n+1)$-dimensional $C^{1}$-graph. Suppose that $C$ is not an open cell, i.e., $d \leq n$.

Suppose first that $C=\Gamma(f)$ where $D$ is a $d$-dimensional $C^{1}$-cell in $R^{n}$ and $f: D \rightarrow$ $R$ is a definable $C^{1}$-function. By induction hypothesis, $D$ is a $d$-dimensional $C^{1}$ graph. After suitably changing the first $n$ coordinates, we may assume that there is a definable $C^{1}$-map $\varphi: \Omega \rightarrow R^{n-d}$ such that $D=\Gamma(\varphi)$. Defining $h: \Omega \rightarrow R^{n-d+1}$ by

$$
h(x)=(\varphi(x), f(x, \varphi(x))) \text { for every } x \in \Omega,
$$


we then have $C=\Gamma(h)$.

Now suppose that $C=(f, g)$ where where $D$ is a $(d-1)$-dimensional $C^{1}$-cell in $R^{n}$ and $f, g: D \rightarrow R$ are definable $C^{1}$-functions with $f<g$. By the same argument as in the above case, we may also assume that $D=\Gamma(\varphi)$ where $\varphi: \Omega \rightarrow R^{n-d}$ is a definable $C^{1}$-map, $\Omega \subseteq R^{d}$ open. Let

$$
E:=\{(x, t) \in \Omega \times R: f(x, \varphi(x))<t<g(x, \varphi(x))\},
$$

a $C^{1}$-cell in $R^{d+1}$, and define $h: E \rightarrow R^{n-d}$ by $h(x, t):=\varphi(x)$ for every $(x, t) \in E$. Define $T: R^{n+1} \rightarrow R^{n+1}$ by

$$
T\left(x_{1}, \ldots, x_{n+1}\right):=\left(x_{1}, \ldots, x_{d}, x_{n+1}, x_{d+1}, \ldots, x_{n}\right) .
$$

Then $T(C)=\Gamma(h)$.

Let $f=\left(f_{1}, \ldots, f_{n}\right): \Omega \rightarrow R^{n}$ be a $C^{1}$-map, where $\Omega$ is an open subset of $R^{d}$, and let $e_{1}, \ldots, e_{d}$ be the standard basis of $R^{d}$. For each $v \in R^{d}$, let $D_{v} f$ denote the directional derivative of $f$ with respect to the vector $v$ and, for $i=1, \ldots, d$,

$$
\frac{\partial f}{\partial x_{i}}:=D_{e_{i}} f=\left(\frac{\partial f_{1}}{\partial x_{i}}, \ldots, \frac{\partial f_{n}}{\partial x_{i}}\right) .
$$

Remark. Let $0<\epsilon<\frac{1}{\sqrt{2}}$ and $f: \Omega \rightarrow R^{n}$ be a definable $C^{1}$-map, where $\Omega$ is an open subset of $R^{d}$, such that $\operatorname{cl}(\Gamma(f))$ is $\epsilon$-flat. Then all partial derivatives of $f$ are bounded by $\frac{\epsilon}{\sqrt{1-\epsilon^{2}}}$.

Proof. Let $a \in \Omega$ and $A: R^{d+n} \rightarrow R^{d+n}$ be the orthogonal projection onto the tangent space $T_{a}(M) \subseteq R^{d+n}$ where $M:=\operatorname{cl}\left(\Gamma(f)\right.$ ). Then, for each $i$ and $w_{i}=\frac{\partial f}{\partial x_{i}}(a)$ (so $\left.\left(e_{i}, w_{i}\right) \in T_{a}(M)\right)$, we have

$$
\frac{\left\|\frac{\partial f}{\partial x_{i}}(a)\right\|^{2}}{1+\left\|\frac{\partial f}{\partial x_{i}}(a)\right\|^{2}}=\frac{\left\|(A-\pi A)\left(e_{i}, w_{i}\right)\right\|^{2}}{\left\|\left(e_{i}, w_{i}\right)\right\|^{2}} \leq \epsilon^{2} .
$$

Therefore, $\left\|\frac{\partial f}{\partial x_{i}}(a)\right\| \leq \frac{\epsilon}{\sqrt{1-\epsilon^{2}}}$.

In the following we will identify each matrix $A \in R^{n \times n}$ (where $n \geq 1$ ) with the $R$-linear map $R^{n} \rightarrow R^{n}$ that is represented by $A$ (with respect to the standard basis of $R^{n}$ ).

Lemma 1.8. Let $E$ be a closed definable subset of $R^{n}$ of constant local dimension $d$ which is $\epsilon$-flat w.r.t. $A \in \mathbb{H}_{n, d}$. Suppose $X$ is a closed definable subset of $R^{n}$ such that $E \backslash X \neq \emptyset$. Then $\operatorname{cl}(E \backslash X)$ is of constant local dimension d and $\epsilon$-flat w.r.t. A.

Proof. We may assume that $A=\pi$ is the natural projection of $R^{n}$ onto its subspace $R^{d} \times\{0\}^{n-d}$. Let $x \in \operatorname{cl}(E \backslash X)$ and let $U$ be an open definable neighborhood of $x$. Then $\emptyset \neq U \cap(E \backslash X)=(U \backslash X) \cap E$. Pick $y \in(U \backslash X) \cap E$. Since $U \backslash X$ is open, there is an open definable neighborhood $V \subseteq U \backslash X$ of $y$ such that $V \cap E$ is of dimension $d$. Then $(U \backslash X) \cap E$ is also of dimension $d$. Therefore, $\operatorname{cl}(E \backslash X)$ is of constant local dimension $d$. 
To show that $\operatorname{cl}(E \backslash X)$ is $\epsilon$-flat, write $E=\bigcup_{i \in I} \operatorname{cl}\left(\Gamma\left(\varphi_{i}\right)\right)$ as the closure of a finite disjoint union of $d$-dimensional $C^{1}$-graphs $\Gamma\left(\varphi_{i}\right)$ in $R^{n}$, each of which is $\epsilon$-flat w.r.t. $A$, where $\varphi_{i}: \Omega_{i} \rightarrow R^{n-d}$ is a definable $C^{1}$-map on a non-empty open definable $\Omega_{i} \subseteq R^{d}$. Obviously, $\operatorname{cl}(E \backslash X)=\bigcup_{i \in I} \operatorname{cl}\left(\Gamma\left(\varphi_{i}\right) \backslash X\right)$. Consider

$$
C_{i}=\pi\left(\operatorname{cl}\left(\Gamma\left(\varphi_{i}\right) \backslash X\right)\right) \subseteq R^{d} \times\{0\}^{n-d} .
$$

We may assume that $C_{i} \neq \emptyset$. Then $C_{i}$ is of constant local dimension $d$. By Proposition 1.3 ,

$$
\operatorname{cl}\left(\Gamma\left(\varphi_{i}\left\lceil\operatorname{int}\left(C_{i}\right)\right)\right)=\operatorname{cl}\left(\Gamma\left(\varphi_{i}\left\lceil C_{i}\right)\right)=\operatorname{cl}\left(\Gamma\left(\varphi_{i}\right) \backslash X\right) .\right.\right.
$$

This completes the proof.

Definition 1.9. By a $\Lambda^{m}$-regular stratification of $R^{n}$ we mean a finite partition $\mathscr{D}$ of $R^{n}$ into $\Lambda^{m}$-regular cells such that each $\partial D(D \in \mathscr{D})$ is a union of sets from $\mathscr{D}$. Given $\epsilon>0$ and definable $E_{1}, \ldots, E_{N} \subseteq R^{n}$, such a $\Lambda^{m}$-regular stratification $\mathscr{D}$ of $R^{n}$ is said to be $\epsilon$-flat if each $D \in \mathscr{D}$ is an $\epsilon$-flat $\Lambda^{m}$-regular cell, and compatible with $E_{1}, \ldots, E_{N}$ if each $E_{i}$ is a union of sets from $\mathscr{D}$.

With these definitions ready, we can now state the main result of [3]:

Theorem 1.10 (Fischer, [3, Theorem 1.4]). Let $E_{1}, \ldots, E_{N}$ be definable subsets of $R^{n}$. Then for each $\epsilon>0$ there exists an $\epsilon$-flat $\Lambda^{m}$-regular stratification of $R^{n}$ which is compatible with $E_{1}, \ldots, E_{N}$.

The following lemma essentially goes back to Gromov [4] (see [6, Section 2]). Recall that a function taking values in $R$ is said to be semidefinite if it is either non-negative on its domain or non-positive on its domain.

Lemma 1.11. Let $h: I \rightarrow R$ be a definable $C^{2}$-function on an interval $I$ in $R$ such that $h, h^{\prime \prime}$ are semidefinite. Let $t \in I$ and $r>0$ such that $[t-r, t+r] \subseteq I$. Then

$$
\left|h^{\prime}(t)\right| \leq \frac{1}{r} \sup \{|h(\xi)|: \xi \in[t-r, t+r]\} .
$$

Proof. Without loss of generality, assume $h^{\prime \prime} \leq 0$ on $I$. By the Mean Value Theorem, since $h^{\prime}$ is decreasing,

$$
\begin{aligned}
& h(t)-h(t-r) \geq r \inf \left\{h^{\prime}(\xi): \xi \in[t-r, t]\right\}=r h^{\prime}(t), \\
& h(t+r)-h(t) \leq r \sup \left\{h^{\prime}(\xi): \xi \in[t, t+r]\right\}=r h^{\prime}(t) .
\end{aligned}
$$

Since $h$ is semidefinite,

$$
|h(t)-h(t-r)|,|h(t+r)-h(t)| \leq \sup \{|h(\xi)|: \xi \in[t-r, t+r]\},
$$

which implies the claim.

For the sake of completeness, we include a proof of the following application of Lemma 1.11 from [3]. In the rest of this section, we fix a non-empty definable open set $\Omega \subseteq R^{d}$, and $\alpha$ ranges over $\mathbb{N}^{d}$. 
Lemma 1.12. Let $f: \Omega \rightarrow R$ be a definable $C^{m+1}$-function such that $D^{\alpha} f$ is semidefinite for $|\alpha| \leq m+1$. Then there is an $L \in \mathbb{Q}^{>0}$ such that

$$
\begin{aligned}
& \left|D^{\alpha} f(u)\right| \leq \frac{L}{r_{0}^{|\alpha|}} \sup \left\{|f(v)|: v \in \Omega,\|v-u\|<r_{0}\right\} \\
& \qquad \quad \text { for }|\alpha| \leq m, u \in \Omega \text {, and } 0<r_{0} \leq d(u, \partial \Omega) .
\end{aligned}
$$

Proof. We proceed by induction on $|\alpha|$. This is trivial if $|\alpha|=0$, so assume we have shown the claim for some $\alpha$ where $|\alpha| \leq m-1$, and let $j \in\{1, \ldots, d\}$. Let $u \in \Omega$, and fix $0<r_{0} \leq d(u, \partial \Omega)$. For each $r \in\left(0, r_{0}\right)$, define $h_{r}:\left[-\frac{r}{2}, \frac{r}{2}\right] \rightarrow R$ by $h_{r}(\xi)=D^{\alpha} f\left(u+\xi e_{j}\right)$ where $e_{j} \in R^{d}$ is the $j$ th standard basis vector of $R^{d}$. For $w \in R^{d}$ with $\|w-u\|<\frac{r}{2}$, we have $w \in \Omega$ and $d(w, \partial \Omega)>\frac{r_{0}}{2}$. By induction hypothesis,

$$
\begin{aligned}
\left|D^{\alpha} f(w)\right| & \leq \frac{L}{\left(r_{0} / 2\right)^{|\alpha|}} \sup \left\{|f(v)|: v \in \Omega,\|v-w\|<\frac{r_{0}}{2}\right\} \\
& \leq \frac{L}{\left(r_{0} / 2\right)^{|\alpha|}} \sup \left\{|f(v)|: v \in \Omega,\|v-w\|<r_{0}\right\} .
\end{aligned}
$$

By Lemma 1.11,

$$
\begin{aligned}
\left|D^{\alpha+e_{j}} f(u)\right|=\left|h_{r}^{\prime}(0)\right| & \leq \frac{1}{r / 2} \sup \left\{|h(\xi)|: \xi \in\left[t-\frac{r}{2}, t+\frac{r}{2}\right]\right\} \\
& \leq \frac{2^{|\alpha|+1} L}{r\left(r_{0}\right)^{|\alpha|}} \sup \left\{|f(v)|: v \in \Omega,\|v-w\|<r_{0}\right\} .
\end{aligned}
$$

Taking $r \rightarrow r_{0}$ yields

$$
\left|D^{\alpha+e_{j}} f(u)\right| \leq \frac{2^{|\alpha|+1} L}{\left(r_{0}\right)^{|\alpha|+1}} \sup \left\{|f(v)|: v \in \Omega,\|v-w\|<r_{0}\right\} .
$$

This finishes the inductive step and this proof.

In [3], Lemma 1.12 is used in the proof of the following proposition:

Proposition 1.13 (Fischer, [3, Proposition 2.1]). Let $f: \Omega \rightarrow R$ be a definable $C^{m}$ function. There are a definable open large subset $U$ of $\Omega$ and some $L \in \mathbb{Q}^{>0}$ with

$$
\begin{aligned}
& \left|D^{\alpha} f(u)\right| \leq \frac{L}{d(u, \partial U)^{|\alpha|}} \sup \{|f(v)|: v \in U,\|u-v\|<d(u, \partial U)\} \\
& \qquad \quad \text { for }|\alpha| \leq m \text { and } u \in U .
\end{aligned}
$$

Here, we follow the same idea and use Lemma 1.12 to prove a modification of Proposition 1.13.

Lemma 1.14. Let $f_{1}, \ldots, f_{k}: \Omega \rightarrow R$ be continuous definable functions. There is $a \Lambda^{m}$-regular stratification $\mathscr{D}$ of $R^{d}$ compatible with $\Omega$ and some $L \in \mathbb{Q}^{>0}$ with the 
following property: for each $D \in \mathscr{D}^{\circ}$ which is contained in $\Omega$, each $f_{i} \uparrow D$ is $C^{m}$ and

$$
\begin{aligned}
& \left|D^{\alpha} f_{i}(u)\right| \leq \frac{L}{d(u, \partial D)^{|\alpha|}} \sup \left\{\left|f_{i}(v)\right|: v \in D,\|u-v\|<d(u, \partial D)\right\} \\
& \qquad \quad \text { for }|\alpha| \leq m \text { and } u \in D .
\end{aligned}
$$

Proof. By the $C^{m+1}$-Cell Decomposition Theorem, take a cell decomposition $\mathscr{C}$ of $R^{d}$ compatible with $\Omega$ such that $f_{i} \uparrow C$ is $C^{m+1}$ for each $C \in \mathscr{C}^{\circ}$ contained in $\Omega$. For $C \in \mathscr{C}^{o}$ contained in $\Omega$ and $|\alpha| \leq m+1, i \in\{1, \ldots, k\}$, set

$$
C_{i}^{\alpha}=\left\{x \in C: D^{\alpha} f_{i}(x)>0\right\}
$$

Next, apply Theorem 1.10 to obtain a $\Lambda^{m}$-regular stratification $\mathscr{D}$ of $R^{d}$ compatible with all $C_{i}^{\alpha}$ 's and all $C \in \mathscr{C}^{\circ}$. By Lemma 1.12, $\mathscr{D}$ has the required properties.

Corollary 1.15. Let $f_{1}, \ldots, f_{k}: \Omega \rightarrow R$ be Lipschitz definable functions. There is a $\Lambda^{m}$-regular stratification $\mathscr{D}$ of $R^{d}$ compatible with $\Omega$ such that, for each $D \in \mathscr{D}^{\circ}$ with $D \subseteq \Omega$, each $f_{i} \uparrow D$ is $\Lambda^{m}$-regular.

Proof. First, by Smooth Cell Decomposition, we may assume that every $f_{i}$ is $C^{1}$. Since all $f_{i}$ are Lipschitz, the partial derivatives $D^{j} f_{i}:=\frac{\partial f_{i}}{\partial x_{j}}$ are bounded for $i=$ $1, \ldots, k$ and $j=1, \ldots, d$. Take $L_{0} \geq 1$ with $\left|D^{j} f_{i}(x)\right| \leq L_{0}$ for all $x \in \Omega$. Then apply Lemma 1.14 to these $D^{j} f_{i}$ 's; therefore, we get a $\Lambda^{m}$-regular stratification $\mathscr{D}$ of $R^{d}$ compatible with $\Omega$ and $L \geq 1$ with the following property: for each $D \in \mathscr{D}^{\circ}$ which is contained in $\Omega$, each $\left(D^{j} f_{i}\right)\left\lceil D\right.$ is $C^{m-1}$ and

$$
\begin{aligned}
\left|D^{\alpha}\left(D^{j} f_{i}\right)(u)\right| & \leq \frac{L}{d(u, \partial D)^{|\alpha|}} \sup \left\{\left|\left(D^{j} f_{i}\right)(v)\right|: v \in D,\|u-v\|<d(u, \partial D)\right\} \\
& \leq \frac{L \cdot L_{0}}{d(u, \partial D)^{|\alpha|}}
\end{aligned}
$$

for $|\alpha| \leq m-1$ and $u \in D$. Since the above inequalities hold for all $j=1, \ldots, d$, for each $D \in \mathscr{D}^{o}$ which is contained in $\Omega$, each $f_{i} \uparrow D$ is $C^{m}$ and

$$
\left|D^{\alpha} f_{i}(u)\right| \leq \frac{L \cdot L_{0}}{d(u, \partial D)^{|\alpha|-1}} \quad \text { for } 1 \leq|\alpha| \leq m \text { and } u \in D
$$

thus each $f_{i} \uparrow D$ is $\Lambda^{m}$-regular.

The previous lemma and corollary immediately imply:

Corollary 1.16. Let $f_{1}, \ldots, f_{k}: \Omega \rightarrow R^{n}$ be continuous definable maps. There is a $\Lambda^{m}$-regular stratification $\mathscr{D}$ of $R^{d}$ compatible with $\Omega$ and some $L \in \mathbb{Q}^{>0}$ such that for each $D \subseteq \Omega$ in $\mathscr{D}^{\circ}$, each restriction $f_{i} \uparrow D$ is $C^{m}$ and

$$
\begin{aligned}
& \left\|D^{\alpha} f_{i}(u)\right\| \leq \frac{L}{d(u, \partial D)^{|\alpha|}} \sup \left\{\left\|f_{i}(v)\right\|: v \in D,\|u-v\|<d(u, \partial D)\right\} \\
& \qquad \quad \text { for }|\alpha| \leq m \text { and } u \in D .
\end{aligned}
$$

Moreover, if $f_{i}$ is Lipschitz, then $f_{i} \uparrow D$ is $\Lambda^{m}$-regular, for each $D \in \mathscr{D}^{\circ}$ with $D \subseteq \Omega$.

In [3], the following useful lemma was shown: 
Lemma 1.17 (Fischer, [3, Lemma 3.8]). Let $0<\epsilon<\frac{1}{32 d^{\frac{3}{2}}}$, and suppose $\Omega$ is an open $\epsilon^{d}$-flat standard $\Lambda^{1}$-regular cell in $R^{d}$. Then, for each pair $a, b \in \Omega$, there exists a definable $C^{1}$-path $\gamma:[0,\|b-a\|] \rightarrow \Omega$ connecting a and $b$ such that $\left\|\gamma^{\prime}(t)-\frac{b-a}{\|b-a\|}\right\|<\epsilon$ for $0<t<\|b-a\|$.

This lemma implies that for every $\epsilon$ small enough, every definable $C^{1}$-map $\Omega \rightarrow R^{n}$ with bounded derivatives, where $\Omega \subseteq R^{d}$ is an $\epsilon$-flat open $\Lambda^{1}$-regular cell, is Lipschitz; more precisely:

Corollary 1.18. Let $0<\epsilon<\frac{1}{32 d^{\frac{3}{2}}}$ and suppose $\Omega$ is an $\epsilon^{d}$-flat standard $\Lambda^{1}$-regular cell in $R^{d}$. Let $f: \Omega \rightarrow R^{n}$ be a definable $C^{1}$-map. Suppose all derivatives of $f$ are bounded by $L \in \mathbb{Q}^{>0}$. Then $f$ is Lipschitz.

Proof. Let $a, b \in \Omega$. By Lemma 1.17, there is a definable $C^{1}$-path $\gamma:[0,\|b-a\|] \rightarrow \Omega$ connecting $a$ and $b$ such that $\left\|\gamma^{\prime}(t)-\frac{b-a}{\|b-a\|}\right\|<\epsilon$ for $0<t<\|b-a\|$. By the Mean Value Theorem,

$$
\begin{aligned}
\|f(b)-f(a)\| & =\|(f \circ \gamma)(\|b-a\|)-(f \circ \gamma)(0)\| \\
& \leq(1+\epsilon)(\sqrt{d+l}) L \cdot\|b-a\| .
\end{aligned}
$$

The next lemma implies that the graph of every definable $C^{1}$-map whose derivatives are bounded by a rational number is $\epsilon$-flat w.r.t. a projection map, for some $\epsilon$ :

Lemma 1.19. Let $M:=\Gamma(f)$ where $f: \Omega \rightarrow R^{n}$ is a definable $C^{1}$-map. Suppose that there is $L \in R^{>0}$ such that $\left\|\frac{\partial f}{\partial x_{j}}\right\| \leq L$ for every $j=1, \ldots, d$. Let $r:=\frac{\sqrt{n} L}{\sqrt{1+n L^{2}}}$. Then $\tau_{M}(M) \subseteq \overline{B_{r}}(\pi)$, where $\pi: R^{d+n} \rightarrow R^{d+n}$ is the natural projection onto $R^{d} \times\{0\}^{n-d}$.

Proof. Let $a \in M$ and let $A: R^{d+n} \rightarrow R^{d+n}$ be the orthogonal projection onto the tangent space $T_{a}(M) \subseteq R^{d+n}$ of $M$ at $a$. If $v \in R^{d}$ with $\|v\|=1$, then $\left\|D_{v} f(a)\right\| \leq$ $\sqrt{n} L$. So for $(v, w) \in T_{a}(M) \subseteq R^{d} \times R^{n}$ where $\|v\|=1$,

$$
\|(A-\pi A)(v, w)\|=\|(v, w)-(v, 0)\|=\|w\|=\left\|D_{v} f(a)\right\| \leq \sqrt{n} L .
$$

Consider $(v, w) \in A\left(R^{d+n}\right)$ where $\|v\|=1$. Since $x \mapsto \frac{x}{1+x}: R^{\geq 0} \rightarrow R^{\geq 0}$ is increasing,

$$
\frac{\|(A-\pi A)(v, w)\|^{2}}{\|(v, w)\|^{2}}=\frac{\left\|D_{v} f(a)\right\|^{2}}{1+\left\|D_{v} f(a)\right\|^{2}} \leq \frac{n L^{2}}{1+n L^{2}} .
$$

For any $(v, w) \in R^{d+n}$, set $\left(v^{\prime}, w^{\prime}\right)=A(v, w)$, so $\left\|\left(v^{\prime}, w^{\prime}\right)\right\| \leq\|(v, w)\|$. Since $A^{2}=A$,

$$
\frac{\|(A-\pi A)(v, w)\|}{\|(v, w)\|}=\frac{\left\|(A-\pi A)\left(v^{\prime}, w^{\prime}\right)\right\|}{\|(v, w)\|} \leq \frac{\left\|(A-\pi A)\left(v^{\prime}, w^{\prime}\right)\right\|}{\left\|\left(v^{\prime}, w^{\prime}\right)\right\|} \leq \frac{\sqrt{n} L}{\sqrt{1+n L^{2}}} .
$$

Hence $\delta(A, \pi) \leq \frac{\sqrt{n} L}{\sqrt{1+n L^{2}}}$.

We end this section by a technical lemma which will be used in Section 2 .

Lemma 1.20. Let $f_{1}, \ldots, f_{k}: E \rightarrow R$ be definable functions, where $E \subseteq R^{n}$, and let $K \in R^{>0}$. Then there are 
(1) a finite family $\left(E_{\mu}\right)$ of definable sets partitioning $E$ and,

(2) a family $\left(M_{\mu}\right)$ (same index set) of elements of $\left\{K, 2 K, \ldots, 2^{k} K\right\}$, such that, for each $i=1, \ldots, k$ and $\mu$, either $\left|f_{i}\right| \leq M_{\mu}$ on $E_{\mu}$ or $\left|f_{i}\right| \geq 2 M_{\mu}$ on $E_{\mu}$. Proof. Let $\Delta$ be the power set of $\{1, \ldots, k\}$, and for each $\delta \in \Delta$ and $L \in R^{>0}$, set

$$
\Omega(\delta, L)=\left\{\left(y_{1}, \ldots, y_{k}\right) \in R^{k}:\left|y_{i}\right| \leq L \text { if } i \in \delta,\left|y_{i}\right| \geq 2 L \text { if } i \notin \delta\right\} .
$$

By induction on $k$, it is easy to show that

$$
R^{k}=\bigcup\left\{\Omega\left(\delta, 2^{j} K\right): \delta \in \Delta, j=0, \ldots, k\right\} .
$$

Take $f: E \rightarrow R^{k}$ where $f=\left(f_{1}, \ldots, f_{k}\right)$ and set

$$
E_{\delta j}=f^{-1}\left(f(E) \cap \Omega\left(\delta, 2^{j} K\right)\right), M_{\delta j}=2^{j} K \quad \text { for } \delta \in \Delta \text { and } j=0, \ldots, k .
$$

One easily sees that the families $\left(E_{\delta j}\right)$ and $\left(M_{\delta j}\right)$ have the desired property.

\section{Separation}

In this section, we still assume that $\epsilon$ ranges over rational numbers. The following important definition goes back to Malgrange's regularly situated condition (see [7]):

Definition 2.1. Let $P, Q, Z \subseteq R^{n}$ be definable. We say that $P$ and $Q$ are $Z$ separated if there exists some $C \in R^{>0}$ such that

$$
d(x, Q) \geq C d(x, Z) \quad \text { for every } x \in P .
$$

Equivalently, there is a $C^{\prime}>0$ such that

$$
d(x, P)+d(x, Q) \geq C^{\prime} d(x, Z) \quad \text { for every } x \in R^{n} .
$$
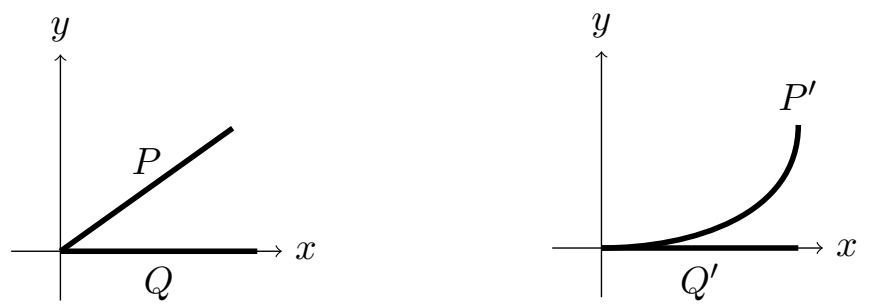

Figure $1 . P$ and $Q$ are $\{(0,0)\}$-separated (left); $P^{\prime}$ and $Q^{\prime}$ are not $\{(0,0)\}$-separated (right).

Our goal in this section is the following theorem, which will become a main tool in the proof of the $\Lambda^{m}$-regular Separation Theorem in the next section:

Theorem 2.2. Let $E$ be a closed definable subset of $R^{n}$ of dimension d. Let $0<\epsilon<$ $\frac{1}{32 n^{\frac{3}{2}}}$ and $A_{1}, \ldots, A_{N} \in \mathbb{H}_{n, d}$ such that $\bigcup_{i=1}^{N} B_{\epsilon}\left(A_{i}\right)=\mathbb{H}_{n, d}$. Fix $\Delta \in \mathbb{Q}$ with

$$
0<\Delta<\sqrt{\frac{(n-d) 2^{d(n-d)} \epsilon^{2}}{1+(n-d) 2^{d(n-d)} \epsilon^{2}}} .
$$

Then there are definable closed subsets $E_{1}, \ldots, E_{N}$ and $B$ of $R^{n}$ such that 
(1) $E=E_{1} \cup \cdots \cup E_{N} \cup B$;

(2) each $E_{i}$ is of dimension d and $\Delta$-flat w.r.t. $A_{i}$, and $B$ is small;

(3) for all $i \neq j$, the intersection $E_{i} \cap E_{j}$ is a small subset of $E$, and there is a small definable set $Z_{i j} \subseteq E_{i} \cup E_{j}$ such that $E_{i}, E_{j}$ are $Z_{i j}$-separated; and

(4) for every $i$ there is a small definable set $Z_{i} \subseteq E_{i} \cup B$ such that $E_{i}, B$ are $Z_{i}$-separated.

We give the proof of this theorem at the end of the section, after some preparations. The following proposition contains simple but important properties of $Z$-separation which will be repeatedly used throughout this and the next sections. All sets in the statement of the proposition are assumed to be definable.

Proposition 2.3 (Pawłucki, [8, Proposition 2]).

(1) If $P, Q$ are $Z$-separated, $P^{\prime} \subseteq P, Q^{\prime} \subseteq Q$ and $Z \subseteq Z^{\prime}$, then $P^{\prime}, Q^{\prime}$ are $Z^{\prime}$-separated.

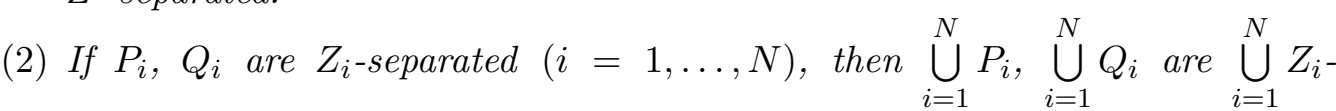
separated.

(3) If $P, Q$ are $S$-separated and $S, Q$ are T-separated, then $P, Q$ are $T$-separated.

Even though we are working in a more general setting than in [8], the proofs of the above proposition and the following lemma and corollaries also work in our context, and for this reason, we omit them.

Lemma 2.4 (Pawłucki, [8, Lemma 6]). Let $f: \Omega \rightarrow R^{n}$ be a definable Lipschitz map on an open set $\Omega \subseteq R^{d}$. Then $\Gamma(f)$ and $\left(R^{d} \backslash \Omega\right) \times R^{n}$ are $\partial \Gamma(f)$-separated.

Corollary 2.5 (Pawłucki, [8, Corollary to Lemma 6]). Let $f: \Omega \rightarrow R^{n}$ be as in the above lemma. Then, for any definable subset $S$ of $\left(R^{d} \backslash \Omega\right) \times R^{n}$, the sets $\Gamma(f)$ and $S$ are $\partial \Gamma(f)$-separated.

Corollary 2.6. Let $C_{1}$ and $C_{2}$ be disjoint open cells in $R^{d}$. Then $C_{1} \times\{0\}^{n}$ and $C_{2} \times\{0\}^{n}$ are $\left(\partial C_{1} \times\{0\}^{n}\right)$-separated.

Using the above, we now show that the separation property behaves nicely for graphs of definable Lipschitz maps and open cells.

Definition 2.7. Let $I=(a, b)$ where $a, b \in R_{ \pm \infty}, a<b$, be an interval, $I \neq R$. Call a definable $C^{1}$-path $\lambda=\left(\lambda_{1}, \ldots, \lambda_{n}\right): I \rightarrow R^{n}$ an admissible arc in $R^{n}$ if for all $i, j$,

(1) $\lambda_{i}$ and $\lambda_{i}^{\prime}$ are of constant sign;

(2) either $\left|\lambda_{i}^{\prime}\right| \geq 1$ on $I$ or $\left|\lambda_{i}^{\prime}\right|<1$ on $I$;

(3) either $\left|\lambda_{i}^{\prime}\right| \leq\left|\lambda_{j}^{\prime}\right|$ on $I$ or $\left|\lambda_{i}^{\prime}\right| \geq\left|\lambda_{j}^{\prime}\right|$ on $I$.

For such an admissible arc $\lambda$, let

$$
\nu(\lambda):=\min \left\{i:\left|\lambda_{i}^{\prime}\right| \geq\left|\lambda_{j}^{\prime}\right| \text { on } I, \text { for all } j\right\} .
$$

We say that an admissible $\operatorname{arc} \lambda$ is fast if $\left|\lambda_{\nu(\lambda)}^{\prime}\right| \geq 1$ on $I$, and slow otherwise.

In [8], it is proved that if $\boldsymbol{R}$ expands the ordered field of real numbers, then the graph $\Gamma$ of a fast admissible arc in $\mathbb{R}^{n}$ and the set $\mathbb{R} \times\{0\}^{n}$ are $\partial \Gamma$-separated. Fortunately, the idea of the proof also works in our more general context. For the 
convenience of the reader, we include detailed proofs of this and other some facts from [8] as warm-up exercises.

Lemma 2.8 (Pawłucki, [8, Lemma 4]). Let $\lambda: I=(a, b) \rightarrow R^{n}$ be a fast admissible arc in $R^{n}$. Let $\widetilde{\lambda}(t)=(t, \lambda(t))$ for $t \in I$, and $T=R \times\{0\}^{n} \subseteq R^{1+n}$. Then, for $t \in I$,

$$
d(\widetilde{\lambda}(t), T) \geq \frac{1}{\sqrt{n+1}}\left\|\tilde{\lambda}(t)-\tilde{\lambda}\left(c_{\lambda}\right)\right\|
$$

where

(1) if $a, b \in R$, then $c_{\lambda}= \begin{cases}a, & \text { if }\left|\lambda_{\nu(\lambda)}\right| \text { is increasing; } \\ b ; & \text { otherwise }\end{cases}$

(2) if $a=-\infty$, then $c_{\lambda}=b$; and

(3) if $b=+\infty$, then $c_{\lambda}=a$.

Proof. Set $\nu=\nu(\lambda)$, and first assume $-\infty<a<b<+\infty$. Replacing $\lambda$ by $-\lambda$ or $\pm \lambda(a+b-t)$ if necessary, we may assume that $\lambda_{\nu}>0$ and $\lambda_{\nu}^{\prime} \geq 1$ on $(a, b)$. Then $c_{\lambda}=a$ and so $\left|\lambda_{\nu}(t)-\lambda_{\nu}(s)\right| \geq|t-s|$. Note that $\lambda\left(c_{\lambda}\right)$ always exists. Hence

$$
d(\widetilde{\lambda}(t), T)=\|\lambda(t)\| \geq \lambda_{\nu}(t) \geq \lambda_{\nu}(t)-\lambda_{\nu}(a) \geq \frac{1}{\sqrt{n+1}}\|\tilde{\lambda}(t)-\tilde{\lambda}(a)\| .
$$

For the rest, we may assume $b=+\infty$. (Replace by $\lambda$ by $t \mapsto \lambda(b-t)$ otherwise.) We see that either $\lambda_{\nu}>0, \lambda_{\nu}^{\prime} \geq 1$ or $\lambda_{\nu}<0, \lambda_{\nu}^{\prime} \leq-1$. The proofs in these two cases are similar to the above case.

Furthermore, given two definable $C^{1}$-maps whose fiberwise differences yield a fast admissible arc, one of the maps being Lipschitz entails that their graphs are separated with respect to the frontier of the graph of the other map:

Lemma 2.9 (Pawłucki, [8, Lemma 5]). Let $C=(\alpha, \beta)$ be an open cell in $R^{d}$ where $\alpha, \beta: D \rightarrow R_{ \pm \infty}$, and not both $\alpha \equiv-\infty$ and $\beta \equiv+\infty$. Let $f, g: C \rightarrow R^{n}$ be $C^{1}$-maps. Assume $f$ is Lipschitz, and for all $u \in D$, the map

$$
\lambda_{u}:(\alpha(u), \beta(u)) \rightarrow R^{n}, \quad \lambda_{u}(y)=g(u, y)-f(u, y)
$$

is a fast admissible arc in $R^{n}$. Then $\Gamma(f), \Gamma(g)$ are $\partial \Gamma(g)$-separated.

Proof. Let $x=(u, y) \in C$. By the above lemma,

$$
d\left(\left(y, \lambda_{u}(y)\right),(\alpha(u), \beta(u)) \times\{0\}^{n}\right) \geq \frac{1}{\sqrt{n+1}}\left\|\left(y, \lambda_{u}(y)\right)-\left(c_{\lambda_{u}}, \lambda_{u}\left(c_{\lambda_{u}}\right)\right)\right\| .
$$

Hence

$$
\begin{aligned}
d\left((x, g(x)-f(x)), \operatorname{cl}(C) \times\{0\}^{n}\right) & =d\left(\left(y, \lambda_{u}(y)\right),(\alpha(u), \beta(u)) \times\{0\}\right) \\
& \geq \frac{1}{\sqrt{n+1}}\left\|\left(y, \lambda_{u}(y)\right)-\left(c_{\lambda_{u}}, \lambda_{u}\left(c_{\lambda_{u}}\right)\right)\right\|,
\end{aligned}
$$

and thus, letting $\bar{f}$ be the continuous extension of $f$ to $\operatorname{cl}(C)$,

$$
\begin{aligned}
& d\left((x, g(x)-f(x)), \operatorname{cl}(C) \times\{0\}^{n}\right) \geq \\
& \frac{1}{\sqrt{n+1}}\left\|(x, g(x)-f(x))-\left(u, c_{\lambda_{u}}, g\left(u, c_{\lambda_{u}}\right)-\bar{f}\left(u, c_{\lambda_{u}}\right)\right)\right\| .
\end{aligned}
$$


For $x \in \operatorname{cl}(C), w \in R^{n}$, set $f^{+}(x, w)=(x, w+\bar{f}(x))$ and $f^{-}(x, w)=(x, w-\bar{f}(x))$. Then $f^{+}$and $f^{-}$are $L$-Lipschitz maps $\operatorname{cl}(C) \times R^{n} \rightarrow R^{d+n}$, for some $L \in R^{>0}$. Let now $x \in C$, and take $\left(x^{\prime}, \bar{f}\left(x^{\prime}\right)\right) \in \Gamma(\bar{f})$ such that

$$
d((x, g(x)), \Gamma(f))=d\left((x, g(x)),\left(x^{\prime}, \bar{f}\left(x^{\prime}\right)\right)\right) .
$$

Then

$$
\begin{aligned}
d((x, g(x)), \Gamma(f)) & =d\left((x, g(x)),\left(x^{\prime}, \bar{g}\left(x^{\prime}\right)\right)\right) \\
& \geq \frac{1}{L} d\left(f^{-}(x, g(x)), f^{-}\left(x^{\prime}, \bar{f}\left(x^{\prime}\right)\right)\right) \\
& =\frac{1}{L} d\left((x, g(x)-f(x)),\left(x^{\prime}, 0\right)\right) \\
& \left.\geq \frac{1}{L} d\left((x, g(x)-f(x)), \operatorname{cl}(C) \times\{0\}^{n}\right)\right) \\
& \geq \frac{1}{L \sqrt{n+1}}\left\|(x, g(x)-f(x))-\left(u, c_{\lambda_{u}}, g\left(u, c_{\lambda_{u}}\right)-\bar{f}\left(u, c_{\lambda_{u}}\right)\right)\right\| \\
& \geq \frac{1}{L^{2} \sqrt{n+1}}\left\|(x, g(x))-\left(u, c_{\lambda_{u}}, \bar{f}\left(u, c_{\lambda_{u}}\right)\right)\right\| \\
& \geq \frac{1}{L^{2} \sqrt{n+1}} d((x, g(x)), \partial \Gamma(g)) .
\end{aligned}
$$

Hence $\Gamma(f), \Gamma(g)$ are $\partial \Gamma(g)$-separated.

The above lemma gives a condition on maps into $R^{n}$ guaranteeing that their graphs are separated. Now, it is quite naturally to ask for a similar condition on a finite number of maps instead of a pair of maps. The following lemma contains such a condition:

Lemma 2.10 (Pawłucki, [8, Lemma 7]). Let $0<\epsilon<\frac{1}{32 d^{\frac{3}{2}}}$ and $\left(\Omega_{\mu}\right)$ be a finite family of disjoint non-empty definable $\epsilon^{d}$-flat open $\Lambda^{1}$-regular cells in $R^{d}$. For every $\mu$, let $f_{\mu \nu}: \Omega_{\mu} \rightarrow R^{n}\left(\nu=1, \ldots, n_{\mu}\right)$ be definable $C^{1}$-maps whose graphs are pairwise disjoint and $M_{\mu} \in \mathbb{Q}^{\geq 1}$ such that, for every $\nu \in\left\{1, \ldots, n_{\mu}\right\}, i \in\{1, \ldots, n\}$, and $j \in\{1, \ldots, d\}$, either $\left|\frac{\partial f_{\mu \nu i}}{\partial x_{j}}\right| \leq M_{\mu}$ on $\Omega_{\mu}$ or $\left|\frac{\partial f_{\mu \nu i}}{\partial x_{j}}\right| \geq 2 M_{\mu}$ on $\Omega_{\mu}$. Set

$$
\begin{aligned}
\Lambda & =\left\{(\mu, \nu):\left|\frac{\partial f_{\mu \nu i}}{\partial x_{j}}\right| \leq M_{\mu} \text { for all } i, j\right\} \\
\Delta_{j} & =\left\{(\mu, \nu):\left|\frac{\partial f_{\mu \nu i}}{\partial x_{j}}\right| \geq 2 M_{\mu} \text { for some } i\right\} \quad(j=1, \ldots, d),
\end{aligned}
$$

and assume that $\Lambda \neq \emptyset$, and $f_{\mu \nu}$ is Lipschitz for each $(\mu, \nu) \in \Lambda$. Consider the definable sets

$$
\begin{aligned}
A & =\bigcup\left\{\Gamma\left(f_{\mu \nu}\right):(\mu, \nu) \in \Lambda\right\}, \\
B_{j} & =\bigcup\left\{\Gamma\left(f_{\mu \nu}\right):(\mu, \nu) \in \Delta_{j}\right\} \quad(j=1, \ldots, d),
\end{aligned}
$$


and let $B=\bigcup_{j=1}^{d} B_{j}$. Then for every definable $B^{\prime} \subseteq B$ and $S \subseteq\left(R^{d} \backslash \bigcup_{\mu} \Omega_{\mu}\right) \times R^{n}$, there is a small definable $Z \subseteq \operatorname{cl}(A) \cup \operatorname{cl}\left(B^{\prime}\right)$ such that $\operatorname{cl}(A), B^{\prime} \cup S$ are $Z$-separated.

Proof. By Corollary 1.18, $f_{\mu \nu}$ is Lipschitz for every $(\mu, \nu) \in \Lambda$. Let $B^{\prime} \subseteq B$ be definable. If, for all $j, \operatorname{cl}(A),\left(B^{\prime} \cap B_{j}\right) \cup S$ are $Z_{j}$-separated for some definable $Z_{j} \subseteq$ $\operatorname{cl}(A) \cup \operatorname{cl}\left(B^{\prime} \cap B_{j}\right)$, then by Proposition 2.3, we're done. Therefore, we may assume that $B^{\prime} \subseteq B_{j}$ and can also assume that $j=d$. Applying the $C^{1}$-Cell Decomposition Theorem, we may further assume that

$$
B^{\prime}=\bigcup\left\{\Gamma\left(h_{\mu^{\prime} \nu^{\prime}}\right):\left|\frac{\partial h_{\mu^{\prime} \nu^{\prime} i}}{\partial x_{d}}\right| \geq 2 M_{\mu^{\prime}}^{\prime} \text { for some } i\right\} \cup T
$$

where $\operatorname{dim}(T)<d,\left(\Omega_{\mu^{\prime}}^{\prime}\right)$ is a finite family of disjoint non-empty definable open subsets of $R^{n}$, and $h_{\mu^{\prime} \nu^{\prime}}: \Omega_{\mu}^{\prime} \rightarrow R^{n}\left(\nu^{\prime}=1, \ldots, n_{\mu^{\prime}}\right)$ are such that

(1) $T \subseteq\left(R^{d} \backslash \bigcup_{\mu^{\prime}} \Omega_{\mu^{\prime}}^{\prime}\right) \times R^{n}$

(2) $\Omega_{\mu^{\prime}}^{\prime} \subseteq \Omega_{\mu}$ for some $\mu$;

(3) $h_{\mu^{\prime} \nu^{\prime}}=f_{\mu \nu} \mid \Omega_{\mu}^{\prime}$ for some $\nu \in\left\{1, \ldots, n_{\mu}\right\}$;

(4) $M_{\mu^{\prime}}^{\prime}=M_{\mu}$ whenever $\Omega_{\mu^{\prime}}^{\prime} \subseteq \Omega_{\mu}$.

So, it is enough to assume that $B^{\prime}=B_{d}$ (and then $Z_{j} \cup T$ works for $\operatorname{cl}(A)$ and $\left.B_{d} \cup T \cup S\right)$. Next, by the Cell Decomposition Theorem and Proposition 1.3, we may assume that $\Omega_{\mu}$ is a cell, and, setting

$$
g_{\mu \nu \nu^{\prime} u}(x)=f_{\mu \nu^{\prime}}(u, x)-f_{\mu \nu}(u, x) \quad \text { where }(\mu, \nu) \in \Lambda,\left(\mu, \nu^{\prime}\right) \in \Delta_{d},
$$

we may assume further that each $g_{\mu \nu \nu^{\prime} u}:\left(\alpha_{\mu}(u), \beta_{\mu}(u)\right) \rightarrow R^{n}$ is an admissible arc in $R^{n}$. Note that $g_{\mu \nu \nu^{\prime} u}$ is fast. By Corollary 2.5 and Lemma 2.9,

$$
Z=\bigcup_{(\mu, \nu) \in \Delta_{d}} \partial \Gamma\left(f_{\mu \nu}\right) \cup \partial A \subseteq \operatorname{cl}(A) \cup \operatorname{cl}(B)
$$

has the required property.

Even though the conditions in the previous lemma look very messy, this lemma turns out to provide a simple and intuitive method for dividing sets into components which are separable with respect to small sets. In order to gain a better understanding, the reader is advised to draw some graphs of continuous function in $\mathbb{R}^{2}$ and try to separate them. To avoid the lengthy conditions in the lemma above, we introduce a more powerful proposition.

Proposition 2.11. Let $E$ be a definable closed subset of $R^{n}$ of dimension $d$ and $C$ be a definable closed subset of $E$ of constant local dimension d. Let $A \in \mathbb{H}_{n, d}$ and $0<\epsilon<\frac{1}{32 n^{\frac{3}{2}}}$, and suppose $C$ is $\epsilon$-flat w.r.t. A. Let $\Delta$ be as in Theorem 2.2. Then there exists a definable closed subset $X$ of $E$ of constant local dimension d such that $C \subseteq X, X$ is $\Delta$-flat w.r.t. A, and for every definable $Y \subseteq E \backslash X$ there is a small definable set $Z \subseteq X \cup \operatorname{cl}(Y)$ such that $X, \operatorname{cl}(Y)$ are $Z$-separated. 
Proof. Without loss of generality, assume $A=\pi$ is the natural projection onto $R^{d} \times$ $\{0\}^{n-d}$. Write $C=\bigcup_{\mu, \nu} \operatorname{cl}\left(\Gamma\left(f_{\mu \nu}\right)\right)$ where $f_{\mu \nu}: \Omega_{\mu} \rightarrow R^{n-d}$, each $\Omega_{\mu}$ is an $\epsilon^{d}$-flat open $\Lambda^{1}$-regular cell in $R^{d}$, and each $\operatorname{cl}\left(\Gamma\left(f_{\mu \nu}\right)\right)$ is $\epsilon$-flat w.r.t. $\pi$. Then for any unit vector $v \in R^{d}$ we have $\frac{\left\|D_{v} f_{\mu \nu}(a)\right\|^{2}}{1+\left\|D_{v} f_{\mu \nu}(a)\right\|^{2}}<\epsilon$ and hence $\left\|D_{v} f_{\mu \nu}(a)\right\|<\frac{\epsilon}{\sqrt{1-\epsilon^{2}}}$. Take a $C^{1}$-cell decomposition $\mathscr{C}$ of $R^{n}$ compatible with the $\Gamma\left(f_{\mu \nu}\right)^{\prime}$ 's, $C$, and $E$. Then $\pi(\mathscr{C})$ is a cell decomposition of $R^{d}$. (Here we identify $R^{d} \times\{0\}^{n-d}$ with $R^{d}$ in the natural way.) Let

$$
\mathscr{C}^{\prime}=\left\{D^{\prime} \in \pi(\mathscr{C})^{o}: D^{\prime} \subseteq \Omega_{\mu} \text { for some } \mu\right\}
$$

Take

$$
E_{1}:=\bigcup_{D^{\prime} \in \mathscr{C}^{\prime}} E \cap \pi^{-1}\left(D^{\prime}\right), \quad E_{2}:=E \backslash E_{1},
$$

and write $E_{1}=\bigcup_{\left(\mu^{\prime}, \nu^{\prime}\right)} \Gamma\left(F_{\mu^{\prime} \nu^{\prime}}\right)$ where $F_{\mu^{\prime} \nu^{\prime}}: \Omega_{\mu^{\prime}}^{\prime} \rightarrow R^{n-d}$ are $C^{1}$.

Claim. $C \subseteq \operatorname{cl}\left(E_{1}\right)$.

Proof of the claim. Let $c \in C$. Since $C$ is of constant local dimension $d, U \cap C$ is of dimension $d$ for any neighborhood $U$ of $c$ small enough. Let $U$ be such a neighborhood of $c$. Then $\pi(U \cap C)$ is also of dimension $d$ (because $C$ is a finite union of graphs). Therefore, there is $D \in \mathscr{C}^{\prime}$ such that $D \cap \pi(U \cap C)$ is of dimension $d$, which implies that $E_{1} \cap U$ is non-empty. Since this holds for all sufficiently small neighborhoods $U$ of $c$, we obtain $c \in \operatorname{cl}\left(E_{1}\right)$.

Let $K=\frac{\epsilon}{1-\epsilon^{2}}$ and $s=d(n-d)$. For each $\mu^{\prime}, \nu^{\prime}$, applying Lemma 1.20 to $\frac{\partial F_{\mu^{\prime} \nu^{\prime}}}{\partial x_{j}}$ $(i \in\{1, \ldots, n-d\}, j \in\{1, \ldots, d\})$, we can decompose $\Omega_{\mu^{\prime}}^{\prime}=\bigcup_{\lambda} B_{\mu^{\prime} \lambda}$ such that for each $\lambda$, there is $K \leq M_{\mu^{\prime} \lambda} \leq 2^{s} K$ such that for each $i, j$, either

$$
\left|\frac{\partial F_{\mu^{\prime} \nu^{\prime} i}}{\partial x_{j}}\right| \leq M_{\mu^{\prime} \lambda} \text { on } B_{\mu^{\prime} \lambda} \quad \text { or } \quad\left|\frac{\partial F_{\mu^{\prime} \nu^{\prime} i}}{\partial x_{j}}\right| \geq 2 M_{\mu^{\prime} \lambda} \text { on } B_{\mu^{\prime} \lambda} \text {. }
$$

By the $\Lambda^{m}$-regular Stratification Theorem and Proposition 1.3, we may assume that the $\Omega_{\mu^{\prime}}^{\prime}$ 's are $\epsilon$-flat and, for each $i, j$, either

$$
\left|\frac{\partial F_{\mu^{\prime} \nu^{\prime} i}}{\partial x_{j}}\right| \leq M_{\mu^{\prime}} \text { on } \Omega_{\mu^{\prime}}^{\prime} \quad \text { or } \quad\left|\frac{\partial F_{\mu^{\prime} \nu^{\prime} i}}{\partial x_{j}}\right| \geq 2 M_{\mu^{\prime}} \text { on } \Omega_{\mu^{\prime}}^{\prime}
$$

Since $\Omega_{\mu^{\prime}}^{\prime}$ is $\epsilon$-flat, by Lemma $1.17, F_{\mu^{\prime} \nu^{\prime}}$ is Lipschitz for each $\nu^{\prime}$ such that for all $i$ and $j,\left|\frac{\partial F_{\mu^{\prime} \nu^{\prime} i}}{\partial x_{j}}\right| \leq M_{\mu^{\prime}}$ on $\Omega_{\mu^{\prime}}^{\prime}$. Let

$$
\begin{aligned}
\Lambda & :=\left\{\left(\mu^{\prime}, \nu^{\prime}\right):\left|\frac{\partial F_{\mu^{\prime} \nu^{\prime} i}}{\partial x_{j}}\right| \leq M_{\mu^{\prime}} \text { for all } i, j\right\}, \\
\Delta_{j} & :=\left\{\left(\mu^{\prime}, \nu^{\prime}\right):\left|\frac{\partial F_{\mu^{\prime} \nu^{\prime} i}}{\partial x_{j}}\right| \geq 2 M_{\mu^{\prime}} \text { for some } i\right\} \quad(j=1, \ldots, d),
\end{aligned}
$$


be similar to the $\Lambda$ and $\Delta_{j}$ in Lemma 2.10, and set

$$
\begin{aligned}
X & :=\bigcup\left\{\operatorname{cl} \Gamma\left(F_{\mu^{\prime} \nu^{\prime}}\right):\left(\mu^{\prime}, \nu^{\prime}\right) \in \Lambda\right\}, \\
B_{j} & :=\bigcup\left\{\Gamma\left(F_{\mu^{\prime} \nu^{\prime}}\right):\left(\mu^{\prime}, \nu^{\prime}\right) \in \Delta_{j}\right\} \quad(j=1, \ldots, d),
\end{aligned}
$$

and $B:=B_{1} \cup \cdots \cup B_{d}$. Since $C \subseteq \operatorname{cl}\left(E_{1}\right), \Lambda \neq \emptyset$. Therefore, by Lemma 1.19, $X$ is $\Delta$-flat w.r.t. $\pi$. Since $\left\|D_{v} f_{\mu \nu}(a)\right\|<\frac{\epsilon}{\sqrt{1-\epsilon^{2}}}$ for any $v \in R^{d}$ and $\frac{\epsilon}{\sqrt{1-\epsilon^{2}}} \leq \frac{\epsilon}{1-\epsilon^{2}}$, we obtain $C \subseteq X$.

To prove the separation condition, let $Y$ be a definable subset of $E$, disjoint from $X$. Applying Lemma 2.10 to

$$
B^{\prime}=Y \cap B, \quad S=Y \cap\left(R^{n} \backslash \bigcup_{\mu^{\prime}}\left(\Omega_{\mu^{\prime}}^{\prime} \times R^{n-d}\right)\right),
$$

we obtain a small definable $Z \subseteq X \cup \operatorname{cl}\left(B^{\prime}\right) \subseteq X \cup \operatorname{cl}(Y)$ such that $X, Y$ are $Z$ separated.

Now, we have all ingredients for the proof of Theorem 2.2.

Proof of Theorem 2.2. By the $C^{1}$-Cell Decomposition Theorem, let $\mathscr{C}$ be a $C^{1}$-cell decomposition of $R^{n}$ compatible with $E$. Let $\mathscr{C}_{d}$ be the set of $C \in \mathscr{C}$ with $\operatorname{dim}(C)=d$, and set

$$
E^{0}:=\bigcup\left\{\operatorname{cl}(C): C \in \mathscr{C}_{d}\right\}, \quad E^{*}=E \backslash E^{0} .
$$

Obviously, $\operatorname{dim}\left(E^{*}\right)<d$. Since each $C \in \mathscr{C}_{d}$ is a $d$-dimensional $C^{1}$-graph, the map $\tau_{C}: \operatorname{cl}(C) \rightarrow \mathbb{H}_{n, d}$ is defined. Apply the $C^{1}$-Cell Decomposition Theorem again, to obtain a $C^{1}$-cell decomposition $\mathscr{D}$ of $R^{n}$ compatible with the $C \in \mathscr{C}_{d}$ of dimension $d$ and the sets $\tau_{C}^{-1}\left(B_{\epsilon}\left(A_{i}\right)\right)$ where $C \in \mathscr{C}_{d}$ and $i=1, \ldots, N$. For $i=1, \ldots, N$, let

$$
\begin{aligned}
\mathscr{D}_{d i} & :=\left\{D \in \mathscr{D}: \operatorname{dim}(D)=d, D \subseteq \tau_{C}^{-1}\left(B_{\epsilon}\left(A_{i}\right)\right) \text { for some } C \in \mathscr{C}_{d}\right\}, \\
E_{i}^{0} & :=\bigcup\left\{\operatorname{cl}(D): D \in \mathscr{D}_{d i}\right\} .
\end{aligned}
$$

Thus $E^{0}=\bigcup_{i=1}^{N} E_{i}^{0}$, and $E_{i}^{0}$ is $\epsilon$-flat w.r.t. $A_{i}$ and closed of constant local dimension $d$. The separation conditions (3) and (4) in Theorem 2.2 in general do not hold for the $E_{i}^{0}$ in place of $E_{i}$. In order to overcome this problem, we will inductively pick definable closed sets $X_{i}, Y_{i}(i=1, \ldots, N)$, such that the following conditions hold:

(1) $X_{1} \supseteq E_{1}^{0}$ and $X_{i} \supseteq E_{i}^{0} \backslash\left(X_{1} \cup \cdots \cup X_{i-1}\right)$,

(2) either $\operatorname{dim}\left(X_{i}\right)=d$ and $X_{i}$ is $\Delta$-flat w.r.t. $A_{i}$ or $X_{i}=\emptyset$,

(3) $Y_{1}=E \backslash X_{1}$ and $Y_{i}=\operatorname{cl}\left(Y_{i-1}\right) \backslash X_{i}$,

(4) $X_{i} \subseteq \operatorname{cl}\left(Y_{i}\right)$

(5) for every definable $Y^{\prime} \subseteq Y_{i}$, there is a small definable set $Z^{\prime} \subseteq X_{i} \cup \operatorname{cl}\left(Y^{\prime}\right)$ such that $X_{i}, \operatorname{cl}\left(Y^{\prime}\right)$ are $Z^{\prime}$-separated.

If $E_{1}^{0}=\emptyset$, let $X_{1}=\emptyset$ and $Y_{1}=E$. If $E_{1}^{0} \neq \emptyset$, by Proposition 2.11, let $X_{1}$ be a definable closed subset of $E$ containing $E_{1}^{0}$, which is of constant local dimension $d$ and $\Delta$-flat w.r.t. $A_{1}$, and $Y_{1}=E \backslash X_{1}$ such that, for $Y^{\prime} \subseteq Y_{1}$, there is a small definable 
set $Z^{\prime} \subseteq X_{1} \cup \operatorname{cl}\left(Y^{\prime}\right)$ such that $X_{1}, \operatorname{cl}\left(Y^{\prime}\right)$ are $Z^{\prime}$-separated. Note that if $Y_{1} \neq \emptyset$, then $\operatorname{dim}\left(Y_{1}\right)=d$.

Next, suppose $X_{1}, Y_{1}, \ldots, X_{i}, Y_{i}$ have been constructed already, where $i<N$. If $E_{i+1}^{0} \backslash\left(X_{1} \cup \cdots \cup X_{i}\right)=\emptyset$, let $X_{i+1}=\emptyset$ and $Y_{i+1}=Y_{i}$. Assume $E_{i+1}^{0} \backslash\left(X_{1} \cup \cdots \cup X_{i}\right) \neq \emptyset$. By Lemma 1.8, $E_{i+1}^{0} \backslash\left(X_{1} \cup \cdots \cup X_{i}\right)$ is of constant local dimension $d$ and $\epsilon$-flat w.r.t. $A_{i+1}$. By Proposition 2.11, $\operatorname{cl}\left(Y_{i}\right)=X_{i+1} \cup Y_{i+1}$ where $Y_{i+1}=\operatorname{cl}\left(Y_{i}\right) \backslash X_{i+1}$ and $X_{i+1}$ is a definable closed subset of $\operatorname{cl}\left(Y_{i}\right)$ of constant local dimension $d$ which is $\Delta$-flat w.r.t. $A_{i+1}$ and contains $E_{i+1}^{0} \backslash\left(X_{1} \cup \cdots \cup X_{i}\right)$, and for every definable $Y^{\prime} \subseteq Y_{i+1}$, there is a small definable set $Z^{\prime} \subseteq X_{i+1} \cup \operatorname{cl}\left(Y^{\prime}\right)$ such that $X_{i+1}, \operatorname{cl}\left(Y^{\prime}\right)$ are $Z^{\prime}$-separated.

By repeating this procedure $N-1$ times, we arrive at the decomposition $E=$ $X_{1} \cup \cdots \cup X_{N} \cup Y_{N}$. Let $i, j \in\{1, \ldots, N\}, i<j$; since $X_{j} \subseteq \operatorname{cl}\left(Y_{i}\right)$, we can pick a small definable $Z_{i j}^{\prime} \subseteq X_{i} \cup X_{j}$ such that $X_{i}, X_{j}$ are $Z_{i j}^{\prime}$-separated. Since $Y_{N} \subseteq \operatorname{cl}\left(Y_{i}\right)$ for all $i$, we can also find a small definable $Z_{i}^{\prime} \subseteq X_{i} \cup Y_{N}$ such that $X_{i}, Y_{N}$ are $Z_{i}^{\prime}$-separated.

Claim. $Y_{N}$ is small.

Proof of the claim. Note $E_{i}^{0} \subseteq X_{1} \cup \cdots \cup X_{i}$ and then $E^{0} \subseteq X_{1} \cup \cdots \cup X_{N}$. Since $Y_{N} \subseteq \operatorname{cl}\left(Y_{i}\right)$ for all $i$, we have

$$
Y_{N} \cap X_{i} \subseteq \operatorname{cl}\left(Y_{i}\right) \cap X_{i}=\operatorname{cl}\left(Y_{i}\right) \backslash Y_{i}=\partial\left(Y_{i}\right),
$$

hence $Y_{N} \cap X_{i}$ is small. Therefore

$$
\begin{aligned}
\operatorname{dim}\left(Y_{N}\right) & =\operatorname{dim}\left(Y_{N} \cap\left(X_{1} \cup \cdots \cup X_{N}\right)\right) \\
& \leq \max \left(\operatorname{dim}\left(Y_{N} \cap X_{1}\right), \ldots, \operatorname{dim}\left(Y_{N} \cap X_{N}\right)\right)<d .
\end{aligned}
$$

Similarly, we also get that $X_{i} \cap X_{j}$ is small.

To finish the proof, let $E_{i}=X_{i}, B=Y_{N} \cup E^{*}, Z_{i j}=Z_{i j}^{\prime}$ and $Z_{i}=Z_{i}^{\prime} \cup E^{*}$.

\section{The $\Lambda^{m}$-Regular Separation Theorem}

We begin with the definition of $\Lambda^{m}$-pancake (which should perhaps more precisely be called "stack of pancakes"):

Definition 3.1. Let $E$ be a definable subset of $R^{n}$ of dimension $d$. We say that $E$ is a $\Lambda^{m}$-pancake if $E$ is a finite disjoint union of graphs of Lipschitz, $\Lambda^{m}$-regular maps $\Omega \rightarrow R^{n-d}$ on a common domain $\Omega$, which is an open $\Lambda^{m}$-regular cell in $R^{d}$.

In this section we show the following:

Theorem 3.2 $\left(\Lambda^{m}\right.$-regular Separation Theorem). Let $E$ be a definable closed subset of $R^{n}$ of dimension d. There is a finite partition $E=M_{1} \cup \cdots \cup M_{s} \cup A$ such that

(1) each $M_{i}$ is a $\Lambda^{m}$-pancake of dimension d in a suitable coordinate system;

(2) A is a small, closed, definable subset of $E$;

(3) for all $i \neq j, \operatorname{cl}\left(M_{i}\right), \operatorname{cl}\left(M_{j}\right)$ are $\partial M_{i}$-separated; and

(4) for each $i, \operatorname{cl}\left(M_{i}\right), A$ are $\partial M_{i}$-separated. 


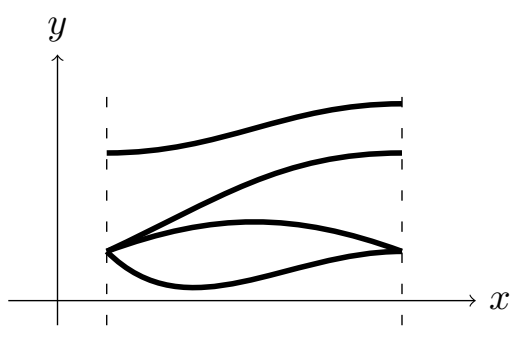

Figure 2. An example of a $\Lambda^{m}$-pancake in $\mathbb{R}^{2}$.

We start by proving a special case of Theorem 3.2, and then the full theorem.

Lemma 3.3. Let $d \leq n$, let $S$ be a definable subset of $R^{n}$ with $\operatorname{dim}(S)<d$, and let $E$ be a finite union of closures of graphs of definable $C^{1}$-maps $\Omega \rightarrow R^{n-d}$ on open subsets $\Omega$ of $R^{d}$, whose derivatives are bounded by a rational. Set $E^{\prime}=E \cup S$, and let $Z$ be a small definable subset of $E^{\prime}$ which contains $S$. Then there is a partition $E^{\prime}=N_{1} \cup \cdots \cup N_{t} \cup B$ where

(1) $N_{i} \subseteq E$ is a $\Lambda^{m}$-pancake in a suitable coordinate system;

(2) $B$ is a small closed definable subset of $E^{\prime}$ containing $Z$;

(3) for all $i \neq j, \operatorname{cl}\left(N_{i}\right), \operatorname{cl}\left(N_{j}\right)$ are $\partial N_{i}$-separated; and

(4) for each $i, \operatorname{cl}\left(N_{i}\right), B$ are $\partial N_{i}$-separated.

Proof. Write $E=\bigcup_{i=1}^{s} \operatorname{cl}\left(\Gamma\left(\varphi_{i}\right)\right)$ where each $\varphi_{i}: \Omega_{i} \rightarrow R^{n-d}$ has bounded derivatives and $\Omega_{i}$ is an open subset of $R^{d}$. First first show that we can assume that each $\varphi_{i}$ is Lipschitz and that $\Omega_{i} \cap \Omega_{j} \neq \emptyset \Rightarrow \Omega_{i}=\Omega_{j}$, for $i, j \in\{1, \ldots, s\}$. To see this, let $0<\epsilon<\frac{1}{32 d^{3 / 2}}$ be a rational number, and by the $\Lambda^{m}$-regular Stratification Theorem 1.10, take an $\epsilon^{d}$-flat $\Lambda^{m}$-regular stratification $\mathscr{D}$ of $R^{d}$ compatible with each $\Omega_{i}$. Since $\varphi_{i}$ has bounded derivatives, $\varphi_{i}\left\lceil D\right.$ is Lipschitz, for each $D \in \mathscr{D}^{o}$, by Corollary 1.18. Now use Proposition 1.3 and replace each $\varphi_{i}$ by the $\varphi_{i}\lceil D$ where $D \in \mathscr{D}^{\circ}$ is a subset of $\Omega_{i}$.

Let $\Theta:=\pi(Z)$, where $\pi$ is the projection $R^{n} \rightarrow R^{d}$ onto the first $d$ coordinates. Then $\operatorname{dim}(\Theta)<d$. By Theorem 1.10 and Corollary 1.16, we can take a $\Lambda^{m}$-regular stratification $\mathscr{D}$ of $R^{d}$ compatible with $\operatorname{cl}(\Omega)$, where $\Omega:=\Omega_{1} \cup \cdots \cup \Omega_{s}$, as well as with the $\Omega_{i}$ and $\Theta$, so that $\varphi_{i} \backslash D$ is $\Lambda^{m}$-regular for each $D \in \mathscr{D}^{o}$ with $D \subseteq \Omega_{i}$. For each $D \in \mathscr{D}^{\circ}$, let

$$
E_{D}=E \cap \pi^{-1}(D), \quad I_{D}=\left\{i \in\{1, \ldots, s\}: D \subseteq \Omega_{i}\right\},
$$

so $E_{D}=\bigcup_{i \in I_{D}} \Gamma\left(\varphi_{i} \mid D\right)$. Note that, by Proposition 1.3, $\bigcup_{D \in \mathscr{D}^{\circ}} \operatorname{cl}\left(E_{D}\right)=E$. Let $N_{1}, \ldots, N_{t}$ be an enumeration of the sets $E_{D}$ with $D \in \mathscr{D}^{\circ}$, and

$$
B:=E^{\prime} \backslash\left(N_{1} \cup \cdots \cup N_{t}\right)=E^{\prime} \backslash \bigcup_{D \in \mathscr{D}^{\circ}} E_{D}=Z \cup \bigcup_{D \in \mathscr{D}^{\circ}} \partial E_{D} ;
$$

thus $B$ is small and $Z \subseteq B$. 
It remains to show the separability conditions. Let $D \in \mathscr{D}^{\circ}$. For $i \in I_{D}$, since $\varphi_{i}\lceil D$ is Lipschitz, by Lemma $2.4, \operatorname{cl}\left(\Gamma\left(\varphi_{i} \backslash D\right)\right)$ and $\left(R^{d} \backslash D\right) \times R^{n-d}$ are $\partial \Gamma\left(\varphi_{i}\lceil D)\right.$-separated. By Proposition 2.3, this yields the claim.

Proof of Theorem 3.2. Let $\epsilon$ be any rational number with $0<\epsilon<\frac{1}{32 n^{3 / 2}}$ and $\Delta$ be as in Theorem 2.2. According to the remark following Definition 1.6, take $A_{1}, \ldots, A_{N} \in$ $\mathbb{H}_{n, d}$ such that $\mathbb{H}_{n, d}=B_{\Delta}\left(A_{1}\right) \cup \cdots \cup B_{\Delta}\left(A_{N}\right)$. By Theorem 2.2, there are closed definable subsets $E_{1}, \ldots, E_{N}, B$ of $E$ such that

(1) $E=E_{1} \cup \cdots \cup E_{N} \cup B$;

(2) each $E_{i}$ is $\Delta$-flat w.r.t. $A_{i} \in \mathbb{H}_{n, d}$, and $B$ is small;

(3) for $i \neq j, E_{i} \cap E_{j}$ is small and there is a small definable $Z_{i j} \subseteq E_{i} \cup E_{j}$ such that $E_{i}, E_{j}$ are $Z_{i j}$-separated;

(4) for each $i$, there is a small definable $Z_{i} \subseteq E_{i} \cup B$ such that $E_{i}, B$ are $Z_{i^{-}}$ separated.

Let $S=Z_{1} \cup \cdots Z_{N} \cup \bigcup_{i \neq j} Z_{i j}$, and for $i \in\{1, \ldots, N\}$ let

$$
S_{i}=S \cup \bigcup_{j \neq i} E_{i} \cap E_{j}, \quad E_{i}^{\prime}=E_{i} \cup S .
$$

By Lemma 3.3, for each $i \in\{1, \ldots, N\}$, there is a partition $E_{i}^{\prime}=N_{1}^{i} \cup \cdots \cup N_{t_{i}}^{i} \cup B^{i}$ such that, with $\alpha, \beta$ ranging over $\left\{1, \ldots, t_{i}\right\}$ :

(1) $N_{\alpha}^{i} \subseteq E_{i}$ is a $\Lambda^{m}$-pancake in a suitable coordinate system;

(2) $B^{i}$ is a small, closed subset of $E_{i}^{\prime}$ containing $S_{i}$;

(3) for $\alpha \neq \beta, \operatorname{cl}\left(N_{\alpha}^{i}\right), \operatorname{cl}\left(N_{\beta}^{i}\right)$ are $\partial N_{\alpha}^{i}$-separated; and,

(4) $\operatorname{cl}\left(N_{\alpha}^{i}\right), B^{i}$ are $\partial N_{\alpha}^{i}$-separated.

Set $A=B^{1} \cup \cdots \cup B^{N} \cup B$. Then

$$
E=\bigcup_{i=1}^{N} \bigcup_{\alpha=1}^{t_{i}}\left(N_{\alpha}^{i} \cup B^{i}\right) \cup B=\left(\bigcup_{i=1}^{N} \bigcup_{\alpha=1}^{t_{i}} N_{\alpha}^{i}\right) \cup A .
$$

In the following, let $i, j \in\{1, \ldots, N\}, \alpha \in\left\{1, \ldots, t_{i}\right\}$ and $\beta \in\left\{1, \ldots, t_{j}\right\}$.

Note that $N_{\alpha}^{i} \cap N_{\beta}^{j}=\emptyset$ for $i \neq j$. We already know that $N_{\alpha}^{i}, N_{\beta}^{i}$ are $\partial N_{\alpha}^{i}$ separated for $\alpha \neq \beta$. Suppose $i \neq j$; we will show that $N_{\alpha}^{i}, N_{\beta}^{j}$ are $\partial N_{\alpha}^{i}$-separated.

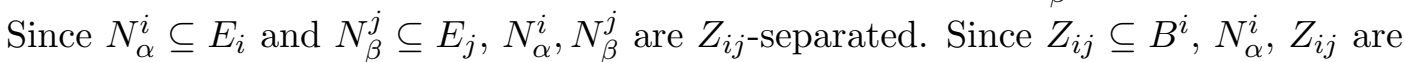
$\partial N_{\alpha}^{i}$-separated. Thus by Proposition $2.3, N_{\alpha}^{i}, N_{\beta}^{j}$ are $\partial N_{\alpha}^{i}$-separated.

Since $N_{\alpha}^{i} \subseteq E_{i}$ and $Z_{i} \subseteq B^{i}, N_{\alpha}^{i}, B$ are $Z_{i}$-separated and $N_{\alpha}^{i}, Z_{i}$ are $\partial N_{\alpha}^{i}$-separated; and so $N_{\alpha}^{i}, B$ are $\partial N_{\alpha}^{i}$-separated. Moreover, as a result of the previous lemma, $N_{\alpha}^{i}, B^{i}$ are $\partial N_{\alpha}^{i}$-separated. Since $N_{\alpha}^{i} \subseteq E_{i}$ and $B_{j} \subseteq E_{j} \cup S, N_{\alpha}^{i}, B^{j}$ are $S$-separated. Since $S \subseteq B^{i}, N_{\alpha}^{i}, S$ are $\partial N_{\alpha}^{i}$-separated. Hence $N_{\alpha}^{i}, B^{j}$ are $\partial N_{\alpha}^{i}$-separated. This implies that $N_{\alpha}^{i}, A$ are $\partial N_{\alpha}^{i}$-separated.

\section{Preliminaries on Whitney Fields}

In this section, we show some basic facts about Whitney fields needed in the proof of our Definable Whitney Extension Theorem in the final two sections of this paper. 
Hestenes' Lemma. The classical incarnation of the first theorem in this section is one of the keys to the study of $C^{m}$-Whitney fields. Here, we give a purely o-minimal proof of this fact. (See [5, Lemma 1] for the classical result.) Recall that $\mathscr{E}^{m}(E)$ denotes the $R$-linear space of definable $C^{m}$-Whitney fields on a definable subset $E$ of $R^{n}$, as defined in the introduction.

Theorem 4.1 (Definable Hestenes' Lemma). Let $\Omega$ be a definable open subset of $R^{n}$. Let $F=\left(F^{\alpha}\right)_{|\alpha| \leq m}$ be a jet of order $m$ on $\Omega$. Let $E$ be a closed definable subset of $\Omega$ such that $F\left\lceil E \in \mathscr{E}^{m}(E)\right.$ and $F \uparrow(\Omega \backslash E) \in \mathscr{E}^{m}(\Omega \backslash E)$. Then $f:=F^{0}$ is $C^{m}$ on $\Omega$ and $D^{\alpha} f=F^{\alpha}$ on $\Omega$. In particular, $F \in \mathscr{E}^{m}(\Omega)$.

Proof. Let $e_{1}, \ldots, e_{n} \in \mathbb{N}^{n}$ be the standard basis of $R^{n}$. Note that if $F^{\beta}$ is $C^{1}$ on $\Omega$ and $\frac{\partial F^{\beta}}{\partial x_{i}}=F^{\beta+e_{i}}$ for $|\beta|<m$ and $i \in\{1, \ldots, n\}$, then $f$ is clearly $C^{m}$ on $\Omega$ and $D^{\alpha} f=F^{\alpha}$ on $\Omega$ for every $|\alpha| \leq m$. Consider the jets $F_{\beta}:=\left(F^{\alpha}\right)_{\alpha \geq \beta}$ of order $m-|\beta|$, for $|\beta| \leq m$. We have $F_{\beta}\left\lceil E \in \mathscr{E}^{m-|\beta|}(E)\right.$ and $F_{\beta} \uparrow(\Omega \backslash E) \in \mathscr{E}^{m-|\beta|}(\Omega \backslash E)$. Hence, it is sufficient to show that $f$ is of class $C^{1}$ on $R^{n}$ and, for every $a \in R^{n}$ and $i \in\{1, \ldots, n\}, \frac{\partial f}{\partial x_{i}}(a)=F^{e_{i}}(a)$; i.e., for every $\epsilon>0$, there is $\delta>0$ such that

$$
\left|f\left(a+t \cdot e_{i}\right)-\left(f(a)+F^{e_{i}}(a) \cdot t\right)\right| \leq \epsilon \cdot|t| \quad \text { for } 0<|t|<\delta .
$$

Let $a \in R^{n}$ and $i \in\{1, \ldots, n\}$. Since $\frac{\partial f}{\partial x_{i}}=F^{e_{i}}$ on $R^{n} \backslash E$ (by the definition of definable $C^{m}$-Whitney fields), we may assume that $a \in E$. Let $\epsilon>0$ be given. For $x, y \in R^{n}$ set

$$
(x, y):=\{x+t \cdot(y-x): t \in(0,1)\} .
$$

By the Cell Decomposition Theorem, there is $\delta_{0}>0$ such that either $\left(a, a+\delta_{0} e_{i}\right)$ is contained in $E$, or in $\Omega \backslash E$. If $\left(a, a+\delta_{0} e_{i}\right) \subseteq E$, then, since $a \in E$ and $F\left\lceil E \in \mathscr{E}^{m}(E)\right.$, there is $0<\delta_{1}<\delta_{0}$ such that

$$
\left|f\left(a+t \cdot e_{i}\right)-\left(f(a)+F^{e_{i}}(a) \cdot t\right)\right| \leq \epsilon \cdot t \quad \text { for } 0<t<\delta_{1},
$$

so (1) holds with $\delta=\delta_{1}$. Now suppose $\left(a, a+\delta_{0} e_{i}\right) \subseteq \Omega \backslash E$. By continuity of $F^{e_{i}}$, we may assume that

$$
\left|F^{e_{i}}(x)-F^{e_{i}}(a)\right|<\epsilon \quad \text { for every } x \in\left(a, a+\delta_{0} e_{i}\right) .
$$

Let $t \in\left(0, \delta_{0}\right)$. Since $f$ is $C^{1}$ on $\Omega \backslash E$ with $\frac{\partial f}{\partial x_{i}}=F^{e_{i}}$ on $\Omega \backslash E$, by the Mean Value Theorem,

$$
\begin{aligned}
\left|f\left(a+t \cdot e_{i}\right)-\left(f(a)+F^{e_{i}}(a) \cdot t\right)\right| & \leq\left|\left(F^{e_{i}}(\xi)-F^{e_{i}}(a)\right) \cdot t\right| \text { some } \xi \in\left(a, a+t \cdot e_{i}\right) \\
& <\epsilon \cdot t .
\end{aligned}
$$

Therefore, there is $\delta_{1}>0$ such that

$$
\left|f\left(a+t \cdot e_{i}\right)-\left(f(a)+F^{e_{i}}(a) \cdot t\right)\right|<\epsilon \cdot t \quad \text { for } 0<t<\delta_{1} .
$$

By the same argument, we can also find $\delta_{2}>0$ such that

$$
\left|f\left(a-t \cdot e_{i}\right)-\left(f(a)+F^{e_{i}}(a) \cdot(-t)\right)\right|<\epsilon \cdot t \quad \text { for } 0<t<\delta_{2} .
$$

Then (1) holds with $\delta=\min \left\{\delta_{1}, \delta_{2}\right\}$. 
Let $E \subseteq R^{n}, E^{\prime} \subseteq R^{n^{\prime}}$ be definable and $\varphi=\left(\varphi_{1}, \ldots, \varphi_{n}\right): U^{\prime} \rightarrow U$ be definable and $C^{m}$, where $U \subseteq R^{n}, U^{\prime} \subseteq R^{n^{\prime}}$ are open definable neighborhoods of $E$, $E^{\prime}$, respectively, such that $\varphi\left(E^{\prime}\right) \subseteq E$. Then $\varphi$ induces an $R$-linear map $F \mapsto \varphi^{*} F: \mathscr{E}^{m}(E) \rightarrow$ $\mathscr{E}^{m}\left(E^{\prime}\right)$ as follows: suppose $a^{\prime} \in E^{\prime}, a=\varphi\left(a^{\prime}\right) \in E$, and view

$$
T_{a}^{m} F=\sum_{|\alpha| \leq m} F^{\alpha}(a) \frac{(x-a)^{\alpha}}{\alpha !}
$$

as an element of the polynomial ring $R\left[x_{1}-a_{1}, \ldots, x_{n}-a_{n}\right]$. Then $\varphi^{*} F$ is the jet of order $m$ on $E^{\prime}$ such that for each $a^{\prime} \in E^{\prime}$, the Taylor polynomial $T_{a^{\prime}}^{m} \varphi^{*} F$ can be obtained by substituting $T_{a^{\prime}}^{m} \varphi_{i} \in R\left[x_{1}^{\prime}-a_{1}^{\prime}, \ldots, x_{n^{\prime}}-a_{n^{\prime}}^{\prime}\right]$ for $x_{i}$ in the polynomial $T_{a}^{m} F$ and dropping the terms of degree $>m$ in $x^{\prime}-a^{\prime}$. It is easy to verify that $\varphi^{*} F$ is a (definable) $C^{m}$-Whitney field on $E^{\prime}$ (the pullback of $F$ under $\varphi$ ).

If $f: U \rightarrow R$ is a definable $C^{m}$-function, then $\varphi^{*}\left(J^{m}(f)\right)=J^{m}(f \circ \varphi)$. Moreover, if $E_{1} \subseteq E, E_{1}^{\prime} \subseteq E^{\prime}$ are definable such that $\varphi\left(E_{1}^{\prime}\right) \subseteq E_{1}$, then

$$
\left(\varphi^{*} F\right)\left\lceil E_{1}^{\prime}=\varphi^{*}\left(F\left\lceil E_{1}\right) \quad \text { for all } F \in \mathscr{E}^{m}(E) .\right.\right.
$$

If $\varphi^{\prime}: U^{\prime \prime} \rightarrow U^{\prime}$ is another definable $C^{m}$-map and $E^{\prime \prime} \subseteq U^{\prime \prime}$ definable with $\varphi\left(E^{\prime \prime}\right) \subseteq E^{\prime}$, then $\left(\varphi \circ \varphi^{\prime}\right)^{*}=\left(\varphi^{\prime}\right)^{*} \circ \varphi^{*}$.

Given a pair $E^{\prime} \subseteq E$ of definable subsets of $R^{n}$, we say that a jet $F$ of order $m$ on $E$ is flat on $E^{\prime}$ if $F\left\lceil E^{\prime}=0\right.$, and we let $\mathscr{E} m\left(E, E^{\prime}\right)$ be the subspace of $\mathscr{E}^{m}(E)$ consisting of the definable $C^{m}$-Whitney fields on $E$ which are flat on $E^{\prime}$.

Proposition 4.2 (Kurdyka \& Pawłucki, [6, Proposition 3]). Let $U$ be a definable open subset of $R^{n}$ and $E$ is a definable closed subset of $U$ such that $\operatorname{cl}(E)$ and $\partial U$ are $(\operatorname{cl}(E) \cap \partial U)$-separated. Let $\varphi: U \rightarrow R^{n}$ be a definable $\Lambda^{m}$-regular map with continuous extension $\bar{\varphi}: \operatorname{cl}(U) \rightarrow R^{n}$ to $\operatorname{cl}(U)$. Let $E^{\prime}$ be a definable closed subset of $R^{n}$ containing $\varphi(E)$ and $F=\left(F^{\alpha}\right)_{|\alpha| \leq m}$ be a jet of order $m$ on $E^{\prime}$ such that, for every $x_{0}^{\prime} \in \bar{\varphi}\left(\partial E^{\prime}\right)$ and $|\alpha| \leq m$,

$$
F^{\alpha}(x)=o\left(d\left(x, \partial E^{\prime}\right)^{m-|\alpha|}\right) \quad \text { as } E^{\prime} \ni x \rightarrow x_{0}{ }^{\prime} .
$$

Then, for any $x_{0} \in \partial E$ and $|\alpha| \leq m$,

$$
\left(\varphi^{*} F\right)^{\alpha}(x)=o\left(d(x, \partial E)^{m-|\alpha|}\right) \quad \text { as } E^{\prime} \ni x \rightarrow x_{0} .
$$

We use this proposition to show:

Corollary 4.3. Let $\Omega \subseteq R^{d}$ be definable and open and $E:=\Omega \times\{0\} \subseteq R^{d+l}$. Suppose that $\varphi: \Omega \times R^{l} \rightarrow R^{d+l}$ is a definable $\Lambda^{m}$-regular map and $\bar{\varphi}: \operatorname{cl}(\Omega) \times R^{l} \rightarrow R^{d+l}$ is the continuous extension of $\varphi$. Assume further that $\bar{\varphi}(\partial E)=\partial(\varphi(E))$. Let $F \in$ $\mathscr{E}^{m}(\operatorname{cl}(\varphi(E)), \partial(\varphi(E)))$. For each $|\alpha| \leq m$, define $\bar{F}^{\alpha}: \operatorname{cl}(E) \rightarrow R$ by

$$
\bar{F}^{\alpha}(x):= \begin{cases}\left(\varphi^{*} F\right)^{\alpha}(x), & \text { if } x \in E \\ 0, & \text { otherwise. }\end{cases}
$$

Let $\bar{\varphi}^{*} F:=\left(\bar{F}^{\alpha}\right)_{|\alpha| \leq m}$. Then $\bar{\varphi}^{*} F \in \mathscr{E} m(\operatorname{cl}(E), \partial E)$. 
Proof. By Proposition 4.2, for every $x_{0} \in \partial \Omega$ and $|\alpha| \leq m$,

$$
\left(\varphi^{*} F\right)^{\alpha}(x, 0)=o\left(d(x, \partial \Omega)^{m-|\alpha|}\right) \quad \text { as } \Omega \ni x \rightarrow x_{0} .
$$

Therefore, $\bar{\varphi}^{*} F$ is a jet of order $m$ on $\operatorname{cl}(E)$. Thus, it is sufficient to show that $\bar{\varphi}^{*} F$ has the $C^{m}$-Whitney field property.

Let $x_{0} \in \operatorname{cl}(\Omega)$ and $\alpha \in \mathbb{N}^{d+l}$ with $|\alpha| \leq m$ and $k:=m-|\alpha|$. For each $\gamma=$ $\left(\gamma_{1}, \ldots, \gamma_{d}\right) \in \mathbb{N}^{d}$ with $|\gamma| \leq k$, define $G_{\alpha}^{\gamma}: R^{\bar{d}} \rightarrow R$ by

$$
G_{\alpha}^{\gamma}(x):= \begin{cases}\left(\varphi^{*} F\right)^{\alpha+\bar{\gamma}}(x, 0), & \text { if } x \in \Omega ; \\ 0, & \text { otherwise }\end{cases}
$$

where $\bar{\gamma}=\left(\gamma_{1}, \ldots, \gamma_{d}, 0, \ldots, 0\right) \in \mathbb{N}^{d+l}$. Let $G_{\alpha}:=\left(G_{\alpha}^{\gamma}\right)_{|\gamma| \leq k}$. Clearly, $G_{\alpha} \mid \Omega \in \mathscr{E}^{k}(\Omega)$ and $G_{\alpha} \uparrow\left(R^{d} \backslash \Omega\right) \in \mathscr{E}^{k}\left(R^{d} \backslash \Omega\right)$. By Hestenes' Lemma, $G_{\alpha}^{0}$ is $C^{m}$ and so $G_{\alpha} \in$ $\mathscr{E}^{k}\left(R^{d}, R^{d} \backslash \Omega\right)$. Therefore,

$$
\begin{aligned}
\left(R_{(x, 0)}^{m}\left(\bar{\varphi}^{*} F\right)\right)^{\alpha}(y, 0) & =\left(R_{x}^{m} G_{\alpha}\right)^{0}(y) \\
& =o\left(\|x-y\|^{k}\right) \\
& =o\left(\|(x, 0)-(y, 0)\|^{m-|\alpha|}\right) \quad \text { as } \operatorname{cl}(\Omega) \ni x, y \rightarrow x_{0} .
\end{aligned}
$$

Since $\alpha$ is arbitrary, $\bar{\varphi}^{*} F$ is a $C^{m}$-Whitney field on $\operatorname{cl}(E)$.

From now on, if all conditions in Corollary 4.3 hold, we denote $\bar{\varphi}^{*} F$ just by $\varphi^{*} F$ for notational simplicity.

The sets $\Delta_{\epsilon}(E)$. For $\epsilon>0$ and definable $E, E^{\prime} \subseteq R^{n}$ with $E^{\prime} \subseteq \operatorname{cl}(E)$, we let

$$
\Delta_{\epsilon}\left(E, E^{\prime}\right):=\left\{x \in R^{n}: d(x, E)<\epsilon d\left(x, E^{\prime}\right)\right\}
$$

and we set $\Delta_{\epsilon}(E):=\Delta_{\epsilon}(E, \partial E)$. See Figure 3 for an example. The following propositions and lemma will be devoted to useful properties of the sets $\Delta_{\epsilon}(E)$.

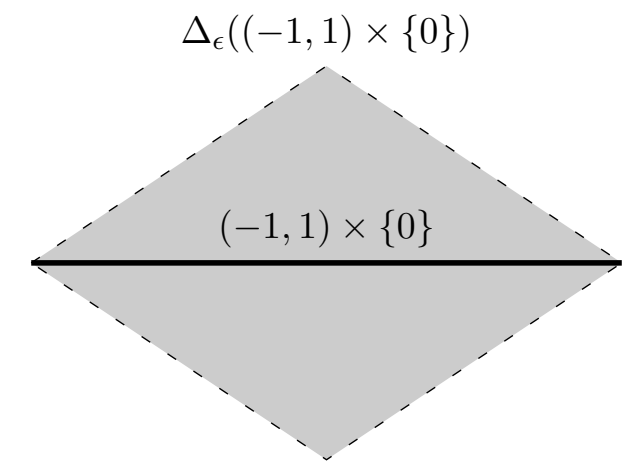

FiguRe 3. $\Delta_{\epsilon}((-1,1) \times\{0\})$ in $\mathbb{R}^{2}$.

Proposition 4.4. Let $\Omega$ be an open cell in $R^{d}$. Then, for each $\epsilon>0$ and each $l$,

$$
\Delta_{\epsilon}\left(\Omega \times\{0\}^{l}\right)=\left\{(x, y) \in \Omega \times R^{l}:|y| \leq \frac{\epsilon}{\sqrt{1-\epsilon^{2}}} d(x, \partial \Omega)\right\} .
$$


We leave the proof of this proposition to the reader. For the next proposition, recall that a subset of $R^{n}$ is locally closed iff its frontier is closed.

Proposition 4.5. Let $E$ be a definable subset of $R^{n}$ which is definably path connected and locally closed. Let $0<\epsilon<1$. Then $\Delta_{\epsilon}(E)$ is also definably path connected.

Proof. It is enough to show that for any $x \in \Delta_{\epsilon}(E)$ we can find a definable path in $\Delta_{\epsilon}(E)$ connecting $x$ and a point in $E$. If $x$ is already in $E$, it is trivial. So, assume $x \in \Delta_{\epsilon}(E) \backslash \operatorname{cl}(E)$. Take $y \in \operatorname{cl}(E)$ with $d(x, y)=d(x, E)$; since $x \in \Delta_{\epsilon}(E)$, we have

$$
d(x, y)=d(x, E)<\epsilon d(x, \partial E) \leq d(x, \partial E),
$$

which implies that $y \notin \partial E$. Let $I=[0, d(x, E)]$ and define $\gamma: I \rightarrow R^{n}$ by $\gamma(t)=$ $x+t \frac{(y-x)}{\|y-x\|}$. Next, we will show that $\gamma(t) \in \Delta_{\epsilon}(E)$ for every $t \in I$ (i.e., $d(\gamma(t), E)<$ $\epsilon d(\gamma(t), \partial E)$.

Claim. $d(\gamma(t), E)=d(\gamma(t), y)$ for all $t \in I$.

Proof of the claim. Pick $z \in E \backslash \partial E$ such that $d(\gamma(t), z)=d(\gamma(t), E)$. If $d(\gamma(t), z)=$ $d(\gamma(t), E)<d(\gamma(t), y)$, then

$$
d(x, z) \leq d(\gamma(t), z)+d(x, \gamma(t))<d(\gamma(t), y)+d(x, \gamma(t))=d(x, y),
$$

which contradicts the minimality of $y$.

Suppose for a contradiction that $\gamma(t) \notin \Delta_{\epsilon}(E)$. Pick $c \in \partial E$ such that $d(\gamma(t), c)=$ $d(\gamma(t), \partial E)$. Then

$$
\begin{aligned}
\epsilon d(x, c) \geq \epsilon d(x, \partial E) & >d(x, E) \\
& =d(x, \gamma(t))+d(\gamma(t), E) \\
& \geq d(x, \gamma(t))+\epsilon d(\gamma(t), \partial E) \\
& =(1-\epsilon) d(x, \gamma(t))+\epsilon[d(x, \gamma(t))+d(\gamma(t), c)] \\
& \geq(1-\epsilon) d(x, \gamma(t))+\epsilon d(x, c),
\end{aligned}
$$

hence $d(x, \gamma(t))<0$, a contradiction. Therefore, $\gamma$ is a definable path in $\Delta_{\epsilon}(E)$ which connects $x$ and $E$.

Proposition 4.6. Let $E=\Gamma(\varphi)$ where $\varphi: \Omega \rightarrow R^{l}$ is definable and Lipschitz and $\Omega$ is an open cell in $R^{d}$. Then there is $\epsilon_{0}>0$ with $\Delta_{\epsilon}(E) \subseteq \Omega \times R^{l}$ for all $0<\epsilon<\epsilon_{0}$.

Proof. Let $L$ be a Lipschitz constant of $\varphi$ and set $\epsilon_{0}=\frac{1}{1+\sqrt{1+L^{2}}}$. Fix $0<\epsilon<\epsilon_{0}$. Let $\bar{\varphi}$ be the Lipschitz extension of $\varphi$ to $\operatorname{cl}(\Omega)$. By the above proposition, it is sufficient to prove that $\Delta_{\epsilon}(E) \cap\left(\partial \Omega \times R^{l}\right)=\emptyset$. Let $x \in \partial \Omega$ and $y \in R^{l}$. Let $a \in \operatorname{cl}(\Omega)$ satisfy 


$$
\begin{aligned}
d((x, y), E)=d((x, y),(a, & \bar{\varphi}(a))) . \text { If }(x, y) \in \Delta_{\epsilon}(E), \text { then } \\
d((x, y), \partial E) & \leq d((x, y),(x, \bar{\varphi}(x))) \\
& \leq d((x, y),(a, \bar{\varphi}(a)))+d((a, \bar{\varphi}(a)),(x, \bar{\varphi}(x))) \\
& \leq d((x, y), E)+\sqrt{1+L^{2}} d(a, x) \\
& \leq d((x, y), E)+\sqrt{1+L^{2}} d((a, \bar{\varphi}(a)),(x, \bar{\varphi}(x))) \\
& =\left(1+\sqrt{1+L^{2}}\right) d((x, y), E) \\
& <\left(1+\sqrt{1+L^{2}}\right) \epsilon d((x, y), \partial E) \\
& \leq d((x, y), \partial E),
\end{aligned}
$$

which is impossible. Thus, $(x, y) \notin \Delta_{\epsilon}(E)$. Since $(x, y)$ is arbitrary in $\partial \Omega \times R^{l}$, we obtain $\Delta_{\epsilon}(E) \cap\left(\partial \Omega \times R^{l}\right)=\emptyset$.

Lemma 4.7. Let $\Omega \subseteq R^{n}$ be open and $E=\bigcup_{i=1}^{N} \Gamma\left(\varphi_{i}\right)$ where each $\varphi_{i}: \Omega \rightarrow R^{l}$ is definable and Lipschitz. Set

$$
\varphi_{i+}(x, y):=\left(x, y+\varphi_{i}(x)\right) \quad \text { for }(x, y) \in \Omega \times R^{l} \text { and } i=1, \ldots, N .
$$

Then

$$
\varphi_{i+}\left(\Delta_{\epsilon}\left(\Omega \times\{0\}^{l}\right)\right) \subseteq \Delta_{2 \epsilon}(E) \quad \text { for all } 0<\epsilon<\frac{1}{\sqrt{2}} \text { and } i \in\{1, \ldots, N\} .
$$

Proof. Let $(x, y) \in \Delta_{\epsilon}(\Omega \times\{0\})$. Then $\|y\|<\frac{\epsilon}{1-\epsilon^{2}} d(x, \partial \Omega)$. Therefore,

$$
\begin{aligned}
d\left(\varphi_{i+}(x, y), E\right) & \leq d\left(\left(x, y+\varphi_{i}(x)\right), E_{i}\right) \\
& \leq d\left(\left(x, y+\varphi_{i}(x)\right),\left(x, \varphi_{i}(x)\right)\right)=\|y\| \\
& <\frac{\epsilon}{\sqrt{1-\epsilon^{2}}} d(x, \partial \Omega) \\
& \leq \frac{\epsilon}{\sqrt{1-\epsilon^{2}}} d\left(\varphi_{i+}(x, y), \partial E\right) \\
& \leq 2 \epsilon \cdot d\left(\varphi_{i+}(x, y), \partial E\right),
\end{aligned}
$$

i.e., $\varphi_{i+}(x, y) \in \Delta_{2 \epsilon}(E)$.

Next, we modify Proposition 6.2 in [9], which is a main step in Pawłucki's version of Whitney's Extension Theorem.

Proposition 4.8. Assume $m \leq q$. Let $E_{i} \supseteq E_{i}^{\prime}(i=1, \ldots, s)$ be definable closed subsets of $R^{n}$ and $C>0$ be a constant such that for any $i, j \in\{1, \ldots, s\}, i \neq j$,

$$
d\left(x, E_{i}\right)+d\left(x, E_{j}\right) \geq C d\left(x, E_{i}^{\prime}\right) \quad \text { for all } x \in R^{n} .
$$

Set $E=E_{1} \cup \cdots \cup E_{N}, E^{\prime}=E_{1}^{\prime} \cup \cdots \cup E_{N}^{\prime}$, and let $F \in \mathscr{E}^{m}\left(E, E^{\prime}\right)$ and $\epsilon \in\left(0, \frac{C}{2}\right)$. Suppose $F\left\lceil E_{i}\right.$ has a definable $C^{m}$-extension $f_{i}$ which is $m$-flat outside $\Delta_{\epsilon}\left(E_{i}, E_{i}^{\prime}\right)$ and $C^{q}$ outside $E_{i}$, for each $i=1, \ldots, s$. Then $f=\sum_{i=1}^{s} f_{i}$ is a definable $C^{m}$-extension of $F$ which is $C^{q}$ outside $E$. 
Proof. It suffices to prove that $\Delta_{\epsilon}\left(E_{i}, E_{i}^{\prime}\right) \cap \Delta_{\epsilon}\left(E_{j}, E_{j}^{\prime}\right)=\emptyset$ for $i \neq j$. Let $x \in$ $\Delta_{\epsilon}\left(E_{i}, E_{i}^{\prime}\right) \cap \Delta_{\epsilon}\left(E_{j}, E_{j}^{\prime}\right)$. Then $d\left(x, E_{i}\right)<\epsilon d\left(x, E_{i}^{\prime}\right)$ and $d\left(x, E_{j}\right)<\epsilon d\left(x, E_{j}^{\prime}\right)$, so

$$
\begin{aligned}
2\left(d\left(x, E_{i}\right)+d\left(x, E_{j}\right)\right) & <2 \epsilon\left(d\left(x, E_{i}^{\prime}\right)+d\left(x, E_{j}^{\prime}\right)\right) \\
& \leq C\left(d\left(x, E_{i}^{\prime}\right)+d\left(x, E_{j}^{\prime}\right)\right) \\
& \leq 2\left(d\left(x, E_{i}\right)+d\left(x, E_{j}\right)\right),
\end{aligned}
$$

a contradiction.

The functions associated to a standard open $\Lambda^{m}$-regular cell. Let $\Omega \subseteq R^{n}$ be a standard open $\Lambda^{m}$-regular cell. Kurdyka and Pawłucki introduced functions $\rho_{j}: \operatorname{cl}(\Omega) \rightarrow R(j=1, \ldots 2 n)$ corresponding to such a cell, which we call the functions associated with $\Omega$, and used them in the proof of their main theorems (see $[6,9])$. These functions also become useful in our construction of definable $C^{m}$-extensions. We define the $\rho_{j}$ by induction on $n$ :

(1) For $n=1$ and $\Omega=(a, b)$,

$$
\rho_{1}(x)=\left\{\begin{array}{ll}
x-a, & \text { if } a \in R, \\
0, & \text { if } a=-\infty,
\end{array} \text { and } \rho_{2}(x)= \begin{cases}b-x, & \text { if } b \in R, \\
0, & \text { if } b=+\infty\end{cases}\right.
$$

(2) Suppose $\Omega^{\prime}$ is a standard open $\Lambda^{m}$-regular cell in $R^{n}$ and $f, g: \Omega^{\prime} \rightarrow R_{ \pm \infty}$ are definable $\Lambda^{m}$-regular functions with

$$
\Omega=\left\{\left(x, x_{n+1}\right) \in \Omega^{\prime} \times R: f(x)<x_{n+1}<g(x)\right\} .
$$

Let $\sigma_{j}(j=1, \ldots, 2 n)$ be the functions associated with $\Omega^{\prime}$. Let $\left(x, x_{n+1}\right) \in$ $\operatorname{cl}(\Omega)$. Set $\rho_{j}\left(x, x_{n+1}\right)=\sigma_{j}(x)$ for $j=1, \ldots, 2 n$ and

$$
\rho_{2 n+1}\left(x, x_{n+1}\right)= \begin{cases}x_{n+1}-f(x) & \text { if } f\left(\Omega^{\prime}\right) \subseteq R, \\ 0 & \text { if } f \equiv-\infty,\end{cases}
$$

and

$$
\rho_{2 n+2}\left(x, x_{n+1}\right)= \begin{cases}g(x)-x_{n+1} & \text { if } g\left(\Omega^{\prime}\right) \subseteq R, \\ 0, & \text { if } g \equiv+\infty .\end{cases}
$$

The proofs of the following facts from [6] (Lemmas 3 and 4) go through in our setting:

Lemma 4.9. Let $\Omega$ be a standard open $\Lambda^{m}$-regular cell in $R^{n}$ and $\rho_{1}, \ldots, \rho_{2 n}$ be the functions associated with $\Omega$.

(1) There is a constant $C>0$ such that

$$
\min _{j} \rho_{j}(x) \leq d(x, \partial \Omega) \leq C \min _{j} \rho_{j}(x) \quad \text { for every } x \in \Omega .
$$

(2) The $\rho_{j}$ are $\Lambda^{m}$-regular.

Pawłucki's proof of Whitney's Extension Theorem in [9] heavily relies on integration of definable functions with respect to parameters, which generally takes us outside our given o-minimal structure $\boldsymbol{R}$, so we cannot immediately follow his proof in our context. In order to overcome this problem, we need to find other definable tools which work in each o-minimal expansion of a real closed ordered field, and one 
of them is the $\Lambda^{m}$-Stratification Theorem. However, this theorem is not sufficient to capture all the necessary information to construct $C^{m}$-extensions for $C^{m}$-Whitney fields. For this reason, the following lemmas are proved, which provide us with some control over the partial derivatives of functions with respect to the boundaries of their domains.

Lemma 4.10. Let $\Omega$ be a definable open subset of $R^{d}$ and $\rho: \Omega \rightarrow R$ be a definable $\Lambda^{m}$-regular function which does not vanish on $\Omega$. Then, for $|\alpha| \leq m$,

$$
D^{\alpha}\left(\frac{1}{\rho}\right)(x)=O\left((\min \{\rho(x), d(x, \partial \Omega)\})^{-|\alpha|-1}\right) \quad \text { as } d(x, \partial \Omega) \rightarrow 0 \text { and } x \in \Omega .
$$

Proof. Let $\lambda_{1}, \ldots, \lambda_{k}$ range over $\mathbb{N}^{d} \backslash\{0\}$. For $\alpha \neq 0$,

$$
D^{\alpha}\left(\frac{1}{\rho}\right)=\sum_{k=1}^{|\alpha|}\left(\sum_{\lambda_{1}+\cdots+\lambda_{k}=\alpha} c_{\lambda_{1} \ldots \lambda_{k}}^{\alpha}\left(D^{\lambda_{1}} \rho\right) \ldots\left(D^{\lambda_{k}} \rho\right)\right) \rho^{-1-k}
$$

where $c_{\lambda_{1} \ldots \lambda_{k}}^{\alpha}$ is an integer. Thus

$$
\begin{aligned}
D^{\alpha}\left(\frac{1}{\rho}\right) & =\sum_{k=1}^{|\alpha|}\left(\sum_{\lambda_{1}+\cdots+\lambda_{k}=\alpha} O\left(d(x, \partial \Omega)^{-\left|\lambda_{1}\right|+1}\right) \cdots O\left(d(x, \partial \Omega)^{-\left|\lambda_{k}\right|+1}\right) \rho^{-1-k}\right. \\
& =O\left(\min \{\rho(x), d(x, \partial \Omega)\}^{-|\alpha|-1}\right)
\end{aligned}
$$

as $d(x, \partial \Omega) \rightarrow 0$ and $x \in \Omega$.

Corollary 4.11. Let $\Omega \subseteq R^{d}$ be an open $\Lambda^{m}$-regular cell, and let $A$ be an orthogonal isomorphism of $R^{d}$ such that $A(\Omega)$ is a standard open $\Lambda^{m}$-regular cell. Let $\rho_{1}, \ldots, \rho_{2 d}: A(\Omega) \rightarrow R$ be the functions associated to $A(\Omega)$. Then, for $|\alpha| \leq m$ and $j=1, \ldots, 2 d$,

$$
D^{\alpha}\left(\frac{1}{\rho_{j}}\right)(x)=O\left(d(x, \partial A(\Omega))^{-|\alpha|-1}\right) \quad \text { as } d(x, \partial A(\Omega)) \rightarrow 0 \text { and } x \in A(\Omega) .
$$

Thus if we let $\nu_{j}=\rho_{j} \circ A$, then

$$
D^{\alpha}\left(\frac{1}{\nu_{j}}\right)(x)=O\left(d(x, \partial \Omega)^{-|\alpha|-1}\right) \quad \text { as } d(x, \partial \Omega) \rightarrow 0 \text { and } x \in \Omega .
$$

Proof. Since each $\rho_{j}$ is $\Lambda^{m}$-regular and $d(x, \partial \Omega) \leq C \rho_{j}(x)$ for some $C>0$, by the above lemma, we're done.

Lemma 4.12. Let $\Omega$ be an open subset of $R^{d}$, let $f: \Omega \times R^{l} \rightarrow R$ and $\rho: \Omega \rightarrow R$ be definable $C^{m}$ functions, and let $t: \Omega \rightarrow R^{>0}$ be definable. Suppose there is $C>0$ such that

$$
t(x) \leq d(x, \partial \Omega) \leq C \cdot \rho(x) \quad \text { for every } x \in \Omega .
$$

Let $\epsilon>0$. Assume, for every $x_{0} \in \partial \Omega$ and $\alpha \in \mathbb{N}^{d}$ with $|\alpha| \leq m$,

$$
D^{\alpha}\left(\frac{1}{\rho}\right)=O\left(t(x)^{-|\alpha|-1}\right) \quad \text { as } x \rightarrow x_{0},
$$

and for $x_{0} \in \partial \Omega$ and $\kappa \in \mathbb{N}^{d+l},|\kappa| \leq m$,

$$
D^{\kappa} f(x, y)=o\left(t(x)^{m-|\kappa|}\right) \quad \text { as } \Delta_{\epsilon}\left(\Omega \times\{0\}^{l}\right) \ni(x, y) \rightarrow\left(x_{0}, 0\right) \text {. }
$$


Fix $i \in\{1, \ldots, l\}$. For every definable $C^{n}$-function $\xi: R \rightarrow R$, where $n \leq m$, set

$$
g_{\xi}(x, y):=\xi\left(\frac{y_{i}}{\rho(x)}\right) f(x, y) \quad \text { for }(x, y) \in \Omega \times R^{l} .
$$

Then for every such $\xi$, $n$, we have, for $|\kappa| \leq n, x_{0} \in \partial \Omega$ :

$$
D^{\kappa} g_{\xi}(x, y)=o\left(t(x)^{n-|\kappa|}\right) \quad \text { as } \Delta_{\epsilon}\left(\Omega \times\{0\}^{l}\right) \ni(x, y) \rightarrow\left(x_{0}, 0\right) .
$$

Proof. Put $h_{0}(x, y)=\frac{y_{i}}{\rho(x)}$ and $h_{\xi}=\xi \circ h_{0}$. By the Leibniz Formula,

$$
D^{\kappa} g_{\xi}=\sum_{\lambda \leq \kappa}\left(\begin{array}{l}
\kappa \\
\lambda
\end{array}\right) D^{\lambda} h_{\xi} D^{\kappa-\lambda} f .
$$

It is enough to check that

$$
D^{\lambda} h_{\xi}(x, y)=O\left(t(x)^{-|\lambda|}\right) \quad \text { as } \Delta_{\epsilon}\left(\Omega \times\{0\}^{l}\right) \ni(x, y) \rightarrow\left(x_{0}, 0\right) .
$$

We proceed by induction on $|\lambda|$. Suppose $|\lambda|=0$. For $(x, y) \in \Delta_{\epsilon}\left(\Omega \times\{0\}^{l}\right)$,

$$
\left|y_{i}\right| \leq d\left((x, y), \Omega \times\{0\}^{l}\right)<\epsilon \cdot d(x, \partial \Omega) \leq \epsilon C \cdot \rho(x)
$$

so $\left|h_{0}(x, y)\right| \leq \epsilon C$. Thus $\xi([-\epsilon C, \epsilon C])$ contains $h_{\xi}\left(\Delta_{\epsilon}\left(\Omega \times\{0\}^{l}\right)\right)$. Since $\xi$ is continuous, the former set is bounded, and hence so is the latter. Therefore $h_{\xi}(x, y)=O(1)$ as $\Delta_{\epsilon}\left(\Omega \times\{0\}^{l}\right) \ni(x, y) \rightarrow\left(x_{0}, 0\right)$.

Assume the claim holds true for some value of $|\lambda| \leq n-1$, where $n \geq 1$. By induction hypothesis,

$$
\begin{aligned}
D^{\lambda+e_{j}} h_{\xi}(x, y) & =\left[D^{\lambda}\left(\frac{\partial h_{\xi}}{\partial x_{j}}\right)\right](x, y) \\
& =\sum_{\mu \leq \lambda}\left(\begin{array}{l}
\lambda \\
\mu
\end{array}\right)\left[D^{\mu}\left(\xi^{\prime} \circ h_{0}\right)\right](x, y)\left[D^{\lambda-\mu}\left(\frac{\partial h_{0}}{\partial x_{j}}\right)\right](x, y) \\
& =\sum_{\mu \leq \lambda}\left(\begin{array}{l}
\lambda \\
\mu
\end{array}\right)\left[D^{\mu} h_{\xi^{\prime}}\right](x, y)\left[D^{\lambda-\mu}\left(\frac{\partial h_{0}}{\partial x_{j}}\right)\right](x, y) \\
& =\sum_{\mu \leq \lambda} O\left(t(x)^{-|\mu|}\right) O\left(t(x)^{-|\lambda|+|\mu|}\right)
\end{aligned}
$$

and so $D^{\lambda+e_{j}} h_{\xi}(x, y)=O\left(t(x)^{-|\lambda|}\right)$ as $\Delta_{\epsilon}\left(\Omega \times\{0\}^{l}\right) \ni(x, y) \rightarrow\left(x_{0}, 0\right)$.

In the rest of this section, we let $0<\epsilon<\frac{1}{\sqrt{2}}$ and $m \leq q$, and we let $\Omega$ be a standard open $\Lambda^{q}$-regular cell in $R^{d}$, with associated functions $\rho_{1}, \ldots, \rho_{2 d}$. We also let $F \in \mathscr{E}^{m}\left(\mathrm{cl}(\Omega) \times\{0\}^{l}, \partial \Omega \times\{0\}^{l}\right)$.

Definition 4.13. Let $\xi: R \rightarrow R$ be a semialgebraic $C^{m}$-function which is 1 in a neighborhood of 0 and 0 outside $(-1,1)$. Define $r_{\epsilon}: R^{d+l} \rightarrow R$ by

$$
r_{\epsilon}(x, y)=\prod_{i=1}^{l} \prod_{j=1}^{2 d} \xi\left(Q_{\epsilon} \frac{y_{i}}{\rho_{j}(x)}\right)
$$

where $Q_{\epsilon}$ is a constant (depending on $\Omega, \epsilon, d$, and $l$ ) large enough so that $r_{\epsilon}$ is $m$-flat outside $\Delta_{\epsilon}\left(\Omega \times\{0\}^{l}\right)$. 
Lemma 4.14. Let $h: \Omega \times R^{l} \rightarrow R$ be definable and $C^{q}$. Suppose, for $\kappa \in \mathbb{N}^{d+l}$ with $|\kappa| \leq m$ and $x_{0} \in \partial \Omega$,

$$
D^{\kappa} h(x, 0)=F^{\kappa}(x, 0) \quad \text { for all } x \in \Omega
$$

and

$$
D^{\kappa} h(x, y)=o\left(d(x, \partial \Omega)^{m-|\kappa|}\right) \quad \text { as } \Delta_{\epsilon}\left(\Omega \times\{0\}^{l}\right) \ni(x, y) \rightarrow\left(x_{0}, 0\right) .
$$

Define $f_{\epsilon}: R^{d+l} \rightarrow R$ by

$$
f_{\epsilon}(x, y)= \begin{cases}r_{\epsilon}(x, y) h(x, y), & \text { if } x \in \Omega \\ 0, & \text { otherwise. }\end{cases}
$$

Then $f_{\epsilon}$ is a definable $C^{m}$-extension of $F$ which is $m$-flat outside $\Delta_{\epsilon}\left(\Omega \times\{0\}^{l}\right)$ and $C^{q}$ outside $\operatorname{cl}(\Omega) \times\{0\}^{l}$.

Proof. Obviously, $f_{\epsilon} \uparrow\left(\Omega \times R^{l}\right)$ is $m$-flat outside $\Delta_{\epsilon}\left(\Omega \times\{0\}^{l}\right)$ and $f_{\epsilon}$ is $C^{q}$ outside $\partial \Omega \times\{0\}^{l}$. First, we will show that $f_{\epsilon}$ extends $F$. Let $x \in \Omega$. Then

$$
f_{\epsilon}(x, 0)=r_{\epsilon}(x, 0) h(x, 0)=F^{0}(x, 0) .
$$

By the Leibniz Formula,

$$
\begin{aligned}
D^{\kappa} f_{\epsilon}(x, y) & =D^{\kappa}\left(r_{\epsilon}(x, y) h(x, y)\right) \\
& =\sum_{\sigma \leq \kappa}\left(\begin{array}{l}
\kappa \\
\sigma
\end{array}\right)\left(D^{\kappa-\sigma} r_{\epsilon}(x, y)\right)\left(D^{\sigma} h(x, y)\right) .
\end{aligned}
$$

Since $\left(D^{\gamma} r_{\epsilon}\right)(x, 0)=0$ if $|\gamma|>0$ and $r_{\epsilon}(x, 0)=1$, we obtain

$$
D^{\kappa} f_{\epsilon}(x, 0)=D^{\kappa} h(x, 0)=F^{\kappa}(x, 0) \text {. }
$$

It remains to show that $f_{\epsilon}$ is actually $C^{m}$ on $R^{d+l}$. Let $y \neq 0 \in R^{l}$. It is enough to find $\delta>0$ such that $(x, y) \notin \Delta_{\epsilon}\left(\Omega \times\{0\}^{l}\right)$ for all $x \in \Omega$ with $d(x, \partial \Omega)<\delta$. Since

$$
(x, y) \notin \Delta_{\epsilon}\left(\Omega \times\{0\}^{l}\right) \Longleftrightarrow|y| \geq \frac{\epsilon}{\sqrt{1-\epsilon^{2}}} d(x, \partial \Omega),
$$

it suffices to pick $\delta=\frac{|y|}{2}$. Therefore, $f_{\epsilon}$ is $C^{m}$ on $R^{d+l} \backslash\left(\partial \Omega \times\{0\}^{l}\right)$. By Corollary 4.11 and Lemma 4.12, $f_{\epsilon}$ is $C^{m}$ on $R^{d+l}$.

Corollary 4.15. For $\beta \in \mathbb{N}^{l}$ with $|\beta| \leq m$, suppose

$$
h^{\beta}: \Omega \times R^{l} \rightarrow R, \quad h^{\beta}(x, y)=F^{(0, \beta)}(x, 0) y^{\beta}
$$

is $C^{q}$ and, for $\kappa \in \mathbb{N}^{d+l}$ with $|\kappa| \leq m$ and $x_{0} \in \partial \Omega$,

$$
D^{\kappa} h^{\beta}(x, y)=o\left(d(x, \partial \Omega)^{m-|\kappa|}\right) \quad \text { as } \Delta_{\epsilon}\left(\Omega \times\{0\}^{l}\right) \ni(x, y) \rightarrow\left(x_{0}, 0\right) .
$$

Define $f_{\epsilon}: R^{d+l} \rightarrow R$ by

$$
f_{\epsilon}(x, y)= \begin{cases}r_{\epsilon}(x, y) \sum_{|\beta| \leq m} \frac{h^{\beta}(x, y)}{\beta !}, & \text { if } x \in \Omega \\ 0, & \text { otherwise. }\end{cases}
$$

Then $f_{\epsilon}$ is a definable $C^{m}$-extension of $F$ which is $m$-flat outside $\Delta_{\epsilon}\left(\Omega \times\{0\}^{l}\right)$ and $C^{q}$ outside $\operatorname{cl}(\Omega) \times\{0\}^{l}$. 
Proof. Clearly, $D^{\kappa}\left(\sum_{|\beta| \leq m} \frac{h^{\beta}(x, 0)}{\beta !}\right)=F^{\kappa}(x, 0)$. By Lemma 4.14, we're done.

The next lemma is a very special case of the main theorem from the introduction. Even though this lemma will not be used later in this paper, it is worth stating here (since this provides an idea of the construction in Section 5).

Lemma 4.16. For $|\alpha| \leq m$ assume that

$$
g^{\alpha}: \Omega \rightarrow R, \quad g^{\alpha}(x)=F^{\alpha}(x, 0)
$$

is $C^{q}$ and that for each $\gamma \in \mathbb{N}^{d}$ with $1 \leq|\gamma| \leq q$, there is some $L>0$ with

$$
\left|D^{\gamma} g^{\alpha}(x)\right| \leq \frac{L}{d(x, \partial \Omega)^{|\gamma|}} \sup \left\{\left|g^{\alpha}(y)\right|: y \in \Omega,\|x-y\|<d(x, \partial \Omega)\right\} \quad \text { for } x \in \Omega \text {. }
$$

Then $F$ has a definable $C^{m}$-extension which is $m$-flat outside $\Delta_{\epsilon}\left(\Omega \times\{0\}^{l}\right)$ and $C^{q}$ outside $\operatorname{cl}(\Omega) \times\{0\}^{l}$.

Proof. For $\beta \in \mathbb{N}^{l}$ with $|\beta| \leq m$, define $h^{\beta}$ as in Corollary 4.15; by this corollary, it is sufficient to prove the following claim:

Claim. For $\kappa=(\sigma, \tau) \in \mathbb{N}^{d} \times \mathbb{N}^{l}$ with $|\kappa| \leq m$ and $x_{0} \in \partial \Omega$,

$$
D^{\kappa} h^{\beta}(x, y)=o\left(d(x, \partial \Omega)^{m-|\kappa|}\right) \quad \text { as } \Delta_{\epsilon}\left(\Omega \times\{0\}^{l}\right) \ni(x, y) \rightarrow\left(x_{0}, 0\right) .
$$

To prove the claim, we may assume $\tau \leq \beta$, since otherwise we simply have $D^{\kappa} h^{\beta}(x, y)=0$. Suppose first that $|\sigma| \leq m-|\beta|$. Then, by Hestenes' Lemma,

$$
D^{\kappa} h^{\beta}(x, y)=\frac{\beta !}{(\beta-\tau) !} F^{(\sigma, \beta)}(x, 0) y^{\beta-\tau}
$$

for every $(x, y) \in \Omega \times R^{l}$. By the definition of $C^{m}$-Whitney fields,

$$
F^{(\sigma, \beta)}(x, 0)=o\left(d(x, \partial \Omega)^{m-|\sigma|-|\beta|}\right) \quad \text { as } x \rightarrow x_{0} .
$$

We have $|y|<\frac{\epsilon}{\sqrt{1-\epsilon^{2}}} d(x, \partial \Omega)$ since $(x, y) \in \Delta_{\epsilon}\left(\Omega \times\{0\}^{l}\right)$, and so (2) follows.

Now suppose $|\sigma|>m-|\beta|$. Then $\sigma=\eta+\gamma$ where $|\eta|=m-|\beta|$. Hence,

$$
\begin{aligned}
D^{\kappa} h^{\beta}(x, y) & =\frac{\beta !}{(\beta-\tau) !} D^{\gamma} g^{(\eta, \beta)}(x) y^{\beta-\tau} \\
& =o\left(d(x, \partial \Omega)^{-|\gamma|}\right) \cdot O\left(d(x, \partial \Omega)^{|\beta|-|\tau|}\right) \\
& =o\left(d(x, \partial \Omega)^{|\beta|-|\tau|-|\gamma|}\right) .
\end{aligned}
$$

Now (2) follows since $|\beta|-|\tau|-|\gamma|=|\beta|-|\tau|-|\sigma|+|\eta|=m-|\kappa|$. This proves the claim.

Corollary 4.17. Assume $\Omega^{\prime} \subseteq R^{d}$ is an open $\Lambda^{q}$-regular cell and $F^{\prime} \in \mathscr{E} m\left(\operatorname{cl}\left(\Omega^{\prime}\right) \times\right.$ $\left.\{0\}^{l}, \partial \Omega^{\prime} \times\{0\}^{l}\right)$. For $|\alpha| \leq m$ define

$$
g^{\alpha}: \Omega^{\prime} \rightarrow R, \quad g^{\alpha}(x)=F^{\alpha}(x, 0) .
$$


Assume $g^{\alpha}$ is $C^{q}$ and, for each $\gamma \in \mathbb{N}^{d}$ with $1 \leq|\gamma| \leq q$, there is some $L>0$ with

$$
\left|D^{\gamma} g^{\alpha}(x)\right| \leq \frac{L}{d\left(x, \partial \Omega^{\prime}\right)^{|\gamma|}} \sup \left\{\left|g^{\alpha}(y)\right|: y \in \Omega^{\prime},\|x-y\|<d\left(x, \partial \Omega^{\prime}\right)\right\} \quad \text { for } x \in \Omega^{\prime} .
$$

Then $F$ has a definable $C^{m}$-extension which is $m$-flat outside $\Delta_{\epsilon}\left(\Omega^{\prime} \times\{0\}^{l}\right)$ and $C^{q}$ outside $\operatorname{cl}\left(\Omega^{\prime}\right) \times\{0\}^{l}$.

Proof. Let $A$ be an orthogonal isomorphism of $R^{d}$ such that $\Omega:=A\left(\Omega^{\prime}\right)$ is a standard open $\Lambda^{q}$-regular cell. Now apply the previous lemma to $\left(A^{-1}\right)^{*} F \in \mathscr{E}^{m}(\operatorname{cl}(\Omega) \times$ $\left.\{0\}^{l}, \partial(\Omega) \times\{0\}^{l}\right)$ in place of $F$.

\section{The First Four Steps}

In this section, we assume $m \leq q$. Pawłucki's construction of an extension operator for $C^{m}$-Whitney fields from [9] can be divided into five steps, depending on the nature of the Whitney field $F$ and its domain $E$ :

Step 1: $E=R^{d} \times\{0\}^{l}$;

Step 2: $E=\operatorname{cl}(\Omega) \times\{0\}^{l}$ where $\Omega$ is an open $\Lambda^{q}$-regular cell and $F$ is flat on $\partial \Omega \times\{0\}^{l}$;

Step 3: $E=\operatorname{cl}\left(E_{0}\right)$ where $E_{0}$ is the graph of Lipschitz $\Lambda^{q}$-regular map on an open $\Lambda^{q}$-regular cell and $F$ is flat on $\partial E_{0}$;

Step 4: $E=\operatorname{cl}\left(E_{0}\right)$ where $E_{0}$ is a $\Lambda^{q}$-regular pancake and $F$ is flat on $\partial E_{0}$;

Step 5: $E$ is any closed definable set.

In this section, we work on the first four steps under the following assumption:

$$
\left\{\begin{array}{l}
\text { For every closed definable set } E \subseteq R^{n} \text { with } \operatorname{dim}(E)<d \text {, every } F \in \\
\mathscr{E}^{m}(E) \text { has a definable } C^{m} \text {-extension which is } C^{q} \text { on } R^{n} \backslash E .
\end{array}\right.
$$

Thus, in the rest of this section we assume that condition $(*)$ holds.

\subsection{Step 1.}

Lemma 5.1. Let $F \in \mathscr{E}^{m}\left(R^{d} \times\{0\}^{l}\right)$. Then $F$ has a definable $C^{m}$-extension which is $C^{q}$ outside $R^{d} \times\{0\}^{l}$.

Proof. For $\beta \in \mathbb{N}^{l}$, define $F_{\beta}:=\left(\widetilde{F}^{(\sigma, \delta)}\right)_{|(\sigma, \delta)| \leq m}$ where

$$
\widetilde{F}^{(\sigma, \delta)}:= \begin{cases}F^{(\sigma, \beta)}, & \text { if } \beta=\delta ; \\ 0, & \text { otherwise. }\end{cases}
$$

Claim. $F_{\beta} \in \mathscr{E}^{m}\left(R^{d} \times\{0\}^{l}\right)$ for every $|\beta| \leq m$.

Proof of the claim. For $x, y \in R^{d}$ and $|(\sigma, \delta)| \leq m$, we have

$$
\begin{aligned}
& \left(R_{(x, 0)}^{m} F_{\beta}(y, 0)\right)^{(\sigma, \delta)} \\
& = \begin{cases}F^{(\sigma, \delta)}(y, 0)-\sum_{|\xi| \leq m-|\beta|-|\sigma|} \frac{F^{(\sigma+\xi, \beta)}(x, 0)}{\xi !(\beta-\gamma) !}(y-x)^{\xi}(0)^{\beta-\delta}, & \text { if } \delta \leq \beta \\
0, & \text { otherwise }\end{cases}
\end{aligned}
$$


with the convention that $0^{0}=1$. For $\delta \neq \beta$, we have $\left(R_{(x, 0)}^{m} F_{\beta}(y, 0)\right)^{(\sigma, \delta)}=0$. Assume $\beta=\delta$. By $C^{m}$-Whitney field property of $F$,

$$
\begin{aligned}
0=\left(R_{(x, 0)}^{m} F(y, 0)\right)^{(\sigma, \beta)} & =F^{(\sigma, \beta)}(y, 0)-\sum_{|(\xi, \eta)| \leq m-|(\sigma, \beta)|} \frac{F^{(\xi, \eta)}(x, 0)}{\xi ! \eta !}(y-x)^{\xi}(0)^{\eta} \\
& =F^{(\sigma, \beta)}(y, 0)-\sum_{|\xi| \leq m-|\beta|-|\sigma|} \frac{F^{(\sigma+\xi, \beta)}(x, 0)}{\xi !}(y-x)^{\xi} \\
& =\left(R_{(x, 0)}^{m} F_{\beta}(y, 0)\right)^{(\sigma, \beta)} .
\end{aligned}
$$

Obviously, $F=\sum_{|\beta| \leq m} F_{\beta}$. Hence, we may assume that $F=F_{\beta}$. By Smooth Cell Decomposition, there is a cell decomposition $\mathscr{C}$ of $R^{d}$ such that, for each $C \in \mathscr{C}$ and $|(\alpha, \beta)| \leq m$, the function $F^{(\alpha, \beta)} \uparrow\left(C \times\{0\}^{l}\right)$ is $C^{q}$. By (*), we may assume the $F$ is flat on $\bigcup_{C \in \mathscr{C} \backslash \mathscr{C}^{\circ}} C \times\{0\}^{l}$.

Let $C \in \mathscr{C}^{\circ}$. By Proposition 4.8 , it is sufficient to find a definable $C^{m}$-extension $f_{C}$ of $F \uparrow\left(\operatorname{cl}(C) \times\{0\}^{l}\right)$ which is $m$-flat outside $\Delta_{\epsilon}\left(C \times\{0\}^{l}\right)$, for some $\epsilon>0$ small enough, and $C^{q}$ outside $\operatorname{cl}(C) \times\{0\}^{l}$. Therefore, we may assume that $F$ is flat on $\left(R^{d} \backslash C\right) \times\{0\}^{l}$ and $F^{(\alpha, \beta)}$ is $C^{q}$ for every $|(\alpha, \beta)| \leq m$. By Lemma 1.14, we may write $\operatorname{cl}(C)=D_{1} \cup \cdots \cup D_{s} \cup B$ where the $D_{i}$ 's are open $\Lambda^{q}$-regular cells and $B=\partial D_{1} \cup \cdots \cup \partial D_{s}$, such that, defining, for $|\alpha| \leq m$,

$$
g^{\alpha}: R^{d} \rightarrow R, \quad g^{\alpha}(x)=F^{\alpha}(x, 0),
$$

there is $L>0$ so that for $\kappa \in \mathbb{N}^{d}$ with $|\kappa| \leq q$ and $u \in D_{i}$, each $g^{\alpha}\left\lceil D_{i}\right.$ is $C^{q}$ and

$$
\left|D^{\kappa} g^{\alpha}(u)\right| \leq \frac{L}{d\left(u, \partial D_{i}\right)^{|\kappa|}} \sup \left\{\left|g^{\alpha}(v)\right|: v \in D_{i},\|u-v\|<d\left(u, \partial D_{i}\right)\right\}
$$

for $u \in D_{i}$.

By $(*)$, let $f_{0}: R^{n} \rightarrow R$ be a definable $C^{m}$-extension of $F \uparrow\left(B \times\{0\}^{l}\right)$ which is $C^{q}$ outside $B \times\{0\}^{l}$, and set

$$
\widetilde{F}:=F-J^{m}\left(f_{0}\right)\left\lceil\left(R^{d} \times\{0\}^{l}\right) \in \mathscr{E}^{m}\left(R^{d} \times\{0\}^{l}\right) .\right.
$$

Clearly,

$$
F_{i}:=\widetilde{F} \uparrow\left(\operatorname{cl}\left(D_{i}\right) \times\{0\}^{l}\right) \in \mathscr{E}^{m}\left(\operatorname{cl}\left(D_{i}\right) \times\{0\}^{l}, \partial D_{i} \times\{0\}^{l}\right) .
$$

By Propostion 4.8, it is sufficient to find a definable $C^{m}$-extension $f_{i}$ for each $F_{i}$ which is $m$-flat outside $\Delta_{\epsilon}\left(D_{i} \times\{0\}^{l}\right)$, for some $\epsilon>0$ small enough, and $C^{q}$ outside $\operatorname{cl}\left(D_{i}\right) \times\{0\}^{l}$. Fix some $i \in\{1, \ldots, s\}$, and let

$$
h_{i}(x, y):=\frac{1}{\beta !} F^{(0, \beta)}(x, 0) y^{\beta}-f_{0}(x, y) .
$$

Obviously, $D^{\kappa} h_{i}(x, 0)=\widetilde{F}^{\kappa}(x, 0)$ for all $x \in D_{i}$ and $|\kappa| \leq m$. By Lemma 4.14 , it is enough to show the following claim: 
Claim. For $\kappa=(\sigma, \tau) \in \mathbb{N}^{d} \times \mathbb{N}^{l}$ with $|\kappa| \leq m$, and $x_{0} \in \partial D_{i}$,

$$
D^{\kappa} h_{i}(x, y)=o\left(d\left(x, \partial D_{i}\right)^{m-|\kappa|}\right) \quad \text { as } \Delta_{\epsilon}\left(D_{i} \times\{0\}^{l}\right) \ni(x, y) \rightarrow\left(x_{0}, 0\right) .
$$

If $x_{0} \in C$, by Taylor's Formula, we're done. Assume $x_{0} \in \partial C$. We will proceed to show the claim by induction on $m-|\kappa|$. First assume $|\kappa|=m$. Clearly,

$$
\left|D^{\kappa} h_{i}(x, y)\right| \leq\left|D^{\kappa}\left(\frac{1}{\beta !} F^{(0, \beta)}(x, 0) y^{\beta}\right)\right|+\left|D^{\kappa} f_{0}(x, y)\right| .
$$

Since $f_{0}$ is $m$-flat at $\left(x_{0}, 0\right)$, we have $D^{\kappa} f_{0}(x, y) \rightarrow 0$ as $(x, y) \rightarrow\left(x_{0}, 0\right)$. Suppose $\tau \leq \beta$ (otherwise, $D^{\kappa}\left(\frac{1}{\beta !} f_{0}^{(0, \beta)}(x, 0) y^{\beta}\right)=0$ ). Then

$$
D^{\kappa}\left(\frac{1}{\beta !} f_{0}^{(0, \beta)}(x, 0) y^{\beta}\right)=\frac{1}{(\beta-\tau) !} D^{\gamma}\left(f_{0}^{(\alpha, \beta)}(x, 0) y^{\beta-\tau}\right)
$$

where $\sigma=\alpha+\gamma$ and $|\alpha|+|\beta|=m$. We have

$$
|\beta|-|\tau|-|\gamma|=|\beta|-|\tau|-|\sigma|+|\alpha|=m-|\tau|-|\sigma|=m-|\kappa|=0 .
$$

Since $F^{(\alpha, \beta)}\left(x_{0}, 0\right)=0$,

$$
s(z):=\sup \left\{\left|F^{(\alpha, \beta)}(x, 0)\right|: x \in D_{i},|x-z|<d\left(z, \partial D_{i}\right)\right\} \rightarrow 0 \quad \text { as } D_{i} \ni z \rightarrow x_{0} .
$$

By (3),

$$
\begin{aligned}
\left|D^{\kappa}\left(\frac{1}{\beta !} f_{0}^{(0, \beta)}(x, 0) y^{\beta}\right)\right| & \leq \frac{L}{d\left(x, \partial D_{i}\right)^{|\gamma|}} s(z)\left(\frac{\epsilon}{\sqrt{1-\epsilon^{2}}} d\left(x, \partial D_{i}\right)\right)^{|\beta|-|\tau|} \\
& =L\left(\frac{\epsilon}{\sqrt{1-\epsilon^{2}}}\right)^{|\beta|-|\tau|} s(z) \\
& \rightarrow 0 \text { as } \Delta_{\epsilon}\left(D_{i} \times\{0\}^{l}\right) \ni(x, y) \rightarrow\left(x_{0}, 0\right) .
\end{aligned}
$$

Next, assume that $|\kappa|<m$ and for every $|\lambda|>|\kappa|$,

$$
D^{\lambda} h_{i}(x, y)=o\left(d\left(x, \partial D_{i}\right)^{m-|\lambda|}\right) \quad \text { as } \Delta_{\epsilon}\left(D_{i} \times\{0\}^{l}\right) \ni(x, y) \rightarrow\left(x_{0}, 0\right) .
$$

Let $(x, y) \in \Delta_{\epsilon}\left(D_{i} \times\{0\}^{l}\right)$. Let $z \in \partial D_{i}$ such that $|x-z|=d\left(x, \partial D_{i}\right)$ and $S$ be the line segment connecting $(x, y)$ and $(z, 0)$. By Proposition 4.4 , we see that $S \subseteq \Delta_{\epsilon}\left(D_{i} \times\{0\}^{l}\right)$. Applying the Mean Value Theorem on $S$, we obtain

$$
\begin{aligned}
\left|D^{\kappa} h(x, y)\right| & \leq \sqrt{d+l} \cdot \sup \left\{\left|D^{\kappa+\lambda} h_{i}(u, w)\right|:|\lambda|=1,(u, w) \in \widetilde{L}\right\} \cdot \sqrt{|x-z|^{2}+|y|^{2}} \\
& \leq(\sqrt{d+l}) \cdot t(x, y) \cdot\left(1+\frac{\epsilon}{\sqrt{1-\epsilon^{2}}}\right) \cdot d\left(x, \partial D_{i}\right)
\end{aligned}
$$

where

$$
\begin{gathered}
t(x, y):=\sup \left\{\left|D^{\kappa+\lambda} h_{i}(u, w)\right|:|\lambda|=1,(u, w) \in \Delta_{\epsilon}\left(D_{i} \times\{0\}^{l}\right),\right. \\
\left.d\left(u, \partial D_{i}\right)<2 d\left(x, \partial D_{i}\right)\right\} .
\end{gathered}
$$

Using the induction hypothesis, we get

$$
\begin{aligned}
D^{\kappa} h_{i}(x, y) & =o\left(d\left(x, \partial D_{i}\right)^{m-|\kappa|-1}\right) \cdot d\left(x, \partial D_{i}\right) \\
& =o\left(d\left(x, \partial D_{i}\right)^{m-|\kappa|}\right) \quad \text { as } \Delta_{\epsilon}\left(D_{i} \times\{0\}^{l}\right) \ni(x, y) \rightarrow\left(x_{0}, 0\right) .
\end{aligned}
$$




\subsection{Step 2.}

Lemma 5.2. Let $\Omega$ be an open $\Lambda^{q}$-regular cell in $R^{d}$, and $F \in \mathscr{E}^{\mathscr{m}}\left(\operatorname{cl}(\Omega) \times\{0\}^{l}, \partial \Omega \times\right.$ $\left.\{0\}^{l}\right)$. Then, for every $\epsilon>0, F$ has a definable $C^{m}$-extension which is $m$-flat outside $\Delta_{\epsilon}\left(\Omega \times\{0\}^{l}\right)$ and $C^{q}$ outside $\operatorname{cl}(\Omega) \times\{0\}^{l}$.

Proof. First, we extend $F$ to $\widetilde{F} \in \mathscr{E}^{m}\left(R^{d} \times\{0\}^{l}\right)$ as follows:

$$
\widetilde{F}^{\alpha}(x, 0)= \begin{cases}F^{\alpha}(x, 0), & \text { if } x \in \Omega ; \\ 0, & \text { otherwise. }\end{cases}
$$

By the above lemma, we can find a definable $C^{m}$-extension $\tilde{f}$ of $\widetilde{F}$. However, $\widetilde{f}$ is possibly not $m$-flat outside $\Delta_{\epsilon}\left(\Omega \times\{0\}^{l}\right)$. In order to guarantee this, we have to slightly modify $\widetilde{f}$. Define

$$
f_{\epsilon}(x, y)= \begin{cases}r_{\epsilon}(x, y) \widetilde{f}(x, y), & \text { if } x \in \Omega \\ 0, & \text { otherwise. }\end{cases}
$$

Here, $r_{\epsilon}$ is as introduced in Definition 4.13. Clearly, $f_{\epsilon}$ is $m$-flat outside $\Delta_{\epsilon}\left(\Omega \times\{0\}^{l}\right)$. Moreover, since $\widetilde{f}$ is $C^{q}$ outside $R^{d} \times\{0\}^{l}$ and $r_{\epsilon}$ is $C^{q}$ on $\Omega \times R^{l}, f_{\epsilon}$ is $C^{q}$ outside $\operatorname{cl}(\Omega) \times\{0\}^{l}$. Since $\tilde{f}$ is $C^{m}$ on $R^{d+l}$, by Corollaries 4.11 and $4.12, f_{\epsilon}$ is $C^{m}$ on $R^{d+l}$.

5.3. Step 3. Let $\varphi: \Omega \rightarrow R^{l}$ be a definable Lipschitz $\Lambda^{q}$-regular map and $\Omega$ be an open $\Lambda^{q}$-regular cell in $R^{d}$. Let $\bar{\varphi}: \operatorname{cl}(\Omega) \rightarrow R^{l}$ be the continuous extension of $\varphi$, and

$$
\begin{array}{ll}
\varphi_{+}: \operatorname{cl}(\Omega) \times R^{l} \rightarrow R^{d+l}, & \varphi_{+}(x, y):=(x, y+\bar{\varphi}(x)), \\
\varphi_{-}: \operatorname{cl}(\Omega) \times R^{l} \rightarrow R^{d+l}, & \varphi_{-}(x, y):=(x, y-\bar{\varphi}(x)) .
\end{array}
$$

To apply Step 2 to $E=\operatorname{cl}(\Gamma(\varphi))$, we first show that for each $C^{m}$-Whitney field on $E$, there is a corresponding $C^{m}$-Whitney field on $\operatorname{cl}(\Omega) \times\{0\}^{l}$.

Let $E_{0}:=\Gamma(\varphi), E:=\operatorname{cl}\left(E_{0}\right)=\Gamma(\bar{\varphi})$, and $F \in \mathscr{E}^{m}\left(E, \partial E_{0}\right)$. Obviously,

$$
\varphi_{+}(\operatorname{cl}(\Omega) \times\{0\})=E, \quad \varphi_{+}(\partial \Omega \times\{0\})=\partial E_{0} .
$$

By Corollary 4.3,

$$
\varphi_{+}^{*} F \in \mathscr{E}^{m}(\operatorname{cl}(\Omega) \times\{0\}, \partial \Omega \times\{0\}) .
$$

Now we show:

Lemma 5.3. Let $E_{0}:=\Gamma(\varphi), E:=\operatorname{cl}\left(E_{0}\right)=\Gamma(\bar{\varphi})$, and $F \in \mathscr{E} m\left(E, \partial E_{0}\right)$. Then, for every $\epsilon>0, F$ has a definable $C^{m}$-extension which is $m$-flat outside $\varphi_{+}\left(\Delta_{\epsilon}\left(\Omega \times\{0\}^{l}\right)\right)$ and $C^{q}$ outside $E$.

Proof. By Proposition 4.6, there is $\epsilon_{0}>0$ such that $\Delta_{\delta}(E) \subseteq \Omega \times R^{l}$ for all $0<\delta<\epsilon_{0}$. Let $\epsilon>0$ be given. We may assume that $\epsilon<\epsilon_{0}$. By Lemma 5.2, take a definable $C^{m}$-extension $f_{-\varphi}$ of $\varphi_{+}^{*} F$ which is $m$-flat outside $\Delta_{\frac{\epsilon}{2}}\left(\Omega \times\{0\}^{n-d}\right)$ and $C^{q}$ outside $\operatorname{cl}(\Omega) \times\{0\}^{n-d}$. Define $f: R^{n} \rightarrow R$ by

$$
f(x, y):= \begin{cases}f_{-\varphi}\left(\varphi_{-}(x, y)\right), & \text { if } x \in \Omega \\ 0, & \text { otherwise }\end{cases}
$$


Since $J^{m}(f) \mid E=\varphi_{-}^{*}\left(\varphi_{+}^{*} F\right)=\left(\varphi_{+} \circ \varphi_{-}\right)^{*} F$ and $\varphi_{+} \circ \varphi_{-}=i d_{\mathrm{cl}(\Omega) \times R^{l}}, J^{m}(f) \mid E=F$. Therefore, $f$ is a $C^{m}$-extension of $F$ which is $m$-flat outside $\widetilde{\varphi}\left(\Delta_{\frac{\epsilon}{2}}\left(\Omega \times\{0\}^{n-d}\right)\right)$ and $C^{q}$ outside $E$.

\subsection{Step 4.}

Lemma 5.4. Let $E_{0}$ be a $\Lambda^{q}$-pancake of dimension d with common domain $\Omega \subseteq R^{d}$, let $E=\operatorname{cl}\left(E_{0}\right)$, and $F \in \mathscr{E}^{m}\left(E, \partial E_{0}\right)$. Then, for every $\epsilon>0, F$ has a definable $C^{m}$-extension which is m-flat outside $\Delta_{\epsilon}\left(E_{0}\right)$ and $C^{q}$ outside $E$.

Proof. Suppose $E=\operatorname{cl}\left(E_{1} \cup \cdots \cup E_{s}\right)$ where $E_{i}=\Gamma\left(\varphi_{i}\right)$ with $\varphi_{i}: \Omega \rightarrow R^{n-d}$ a definable $\Lambda^{q}$-regular Lipschitz map. For each $i \in\{1, \ldots, s\}$, let $\overline{\varphi_{i}}: \operatorname{cl}(\Omega) \rightarrow R^{l}$ be the continuous extension of $\varphi$, and

$$
\begin{array}{ll}
\varphi_{i+}: \operatorname{cl}(\Omega) \times R^{l} \rightarrow R^{d+l}, & \varphi_{i+}(x, y):=\left(x, y+\overline{\varphi_{i}}(x)\right), \\
\varphi_{i-}: \operatorname{cl}(\Omega) \times R^{l} \rightarrow R^{d+l}, & \varphi_{i-}(x, y):=\left(x, y-\overline{\varphi_{i}}(x)\right) .
\end{array}
$$

By Lemma 4.7, it is enough to prove that, for $0<\epsilon<\frac{1}{\sqrt{2}}$, there exists a definable $C^{m}$-extension of $F$ which is $m$-flat outside $\bigcup_{i=1}^{s} \varphi_{i+}\left(\Delta_{\epsilon}\left(\Omega \times\{0\}^{n-d}\right)\right)$ and $C^{q}$ outside $\bigcup_{i=1}^{s} \operatorname{cl}\left(E_{i}\right)$. We show this by induction on $s$. If $s=1$, by Lemma 4.7 , this is just Lemma 5.3. Suppose $s>1$, and the statement is true for $s-1$ in place of $s$.

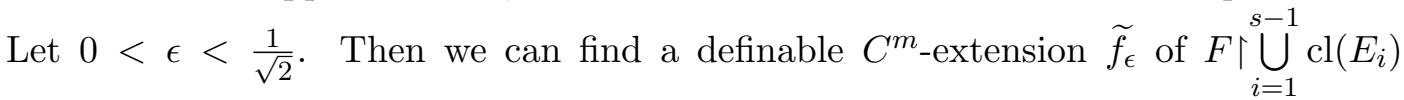
which is $m$-flat outside $\bigcup_{i=1}^{s-1} \varphi_{i+}\left(\Delta_{\epsilon}\left(\Omega \times\{0\}^{n-d}\right)\right)$ and $C^{q}$ outside $\bigcup_{i=1}^{s-1} \operatorname{cl}\left(E_{i}\right)$. Note that $\bigcup_{i=1}^{s-1} \varphi_{i+}\left(\Delta_{\epsilon}\left(\Omega \times\{0\}^{n-d}\right)\right)$ and $\partial \Omega \times R^{n-d}$ are disjoint. After replacing $F$ by $F-J^{m}\left(\tilde{f}_{\epsilon}\right)\lceil E$, we may assume that

$$
F \in \mathscr{E}^{m}\left(\bigcup_{i=1}^{s} \operatorname{cl}\left(E_{i}\right), \bigcup_{i=1}^{s-1} \operatorname{cl}\left(E_{i}\right) \cup \partial E_{s}\right) .
$$

Next, consider $\varphi_{s+}^{*}\left(F\left\lceil\operatorname{cl}\left(E_{s}\right)\right) \in \mathscr{E}^{m}(\operatorname{cl}(\Omega) \times\{0\}, \partial \Omega \times\{0\})\right.$ (by Corollary 4.3.) By Lemma 5.2, let $f$ be a $C^{m}$-extension of $\varphi_{s+}^{*}\left(F\left\lceil\operatorname{cl}\left(E_{s}\right)\right)\right.$ which is $m$-flat outside $\Delta_{\epsilon}(\Omega \times$ $\left.\{0\}^{n-d}\right)$ and $C^{q}$ outside $\operatorname{cl}(\Omega) \times\{0\}^{n-d}$. For $i=1, \ldots, s-1$ and $x \in \Omega$, we define $r_{i}(x):=\left|\varphi_{i}(x)-\varphi_{s}(x)\right|$. Each function $r_{i}: \Omega \rightarrow R^{>0}$ is $\Lambda^{m}$-regular. Let $\xi: R \rightarrow R$ be any semialgebraic $C^{q}$-function which is 1 in a neighborhood of 0 and 0 outside $(-1,1)$. Then, define

$$
g(x, y)= \begin{cases}\prod_{i=1}^{s-1} \prod_{j=1}^{l} \xi\left(\sqrt{l} \frac{y_{j}}{r_{i}(x)}\right) f(x, y), & \text { if } x \in \Omega \\ 0, & \text { otherwise. }\end{cases}
$$

Since $f$ is $C^{m}$, by Lemmas 4.10 and $4.12, g$ is a $C^{m}$-extension of $\varphi_{s+}^{*}\left(F\left\lceil\operatorname{cl}\left(E_{s}\right)\right)\right.$ which is $m$-flat outside $\Delta_{\epsilon}\left(\Omega \times\{0\}^{n-d}\right)$. Moreover, by the choice of $r_{i}$ and $\xi$, we also get 
that $g$ is $m$-flat on $\varphi_{s-}\left(E_{i}\right)$ for all $i=1, \ldots, s-1$. Define $f_{\epsilon}: R^{n} \rightarrow R$ by

$$
f_{\epsilon}(x, y):= \begin{cases}g\left(\varphi_{s-}(x)\right), & \text { if } x \in \Omega \\ 0, & \text { otherwise. }\end{cases}
$$

Obviously, $\operatorname{cl}\left(E_{i}\right)=\varphi_{s+}\left(\varphi_{s-}\left(\operatorname{cl}\left(E_{i}\right)\right)\right)$ for all $i \in\{1, \ldots, s\}$. Thus, $f_{\epsilon}$ is a $C^{m_{-}}$ extension of $F\left\lceil\operatorname{cl}\left(E_{s}\right)\right.$ which is $m$-flat on $\operatorname{cl}\left(E_{i}\right)$ and outside $\varphi_{s+}\left(\Delta_{\epsilon}\left(\Omega \times\{0\}^{n-d}\right)\right)$. Therefore, $f_{\epsilon}$ is a $C^{m}$-extension of $F$ which is $m$-flat outside $\bigcup_{i=1}^{s} \varphi_{i+}\left(\Delta_{\epsilon}\left(\Omega \times\{0\}^{n-d}\right)\right)$. In addition, $f_{\epsilon}$ is $C^{q}$ outside $\bigcup_{i=1}^{s} \operatorname{cl}\left(E_{i}\right)$.

\section{Proof of the Definable Whitney Extension Theorem}

Suppose $m \leq q$. We will prove by induction on $d$ that every $F \in \mathscr{E}^{m}(E)$, where $E$ is a definable closed subset of $R^{n}$ of dimension d, has a definable $C^{m}$-extension which is $C^{q}$ on $R^{n} \backslash E$. When $d=0, E$ is just a finite subset of $R^{n}$; and this case is easy. Suppose $d>0$, and the statement is true for all smaller values of $d$; that is, condition $(*)$ from the previous section holds. Let $E$ be a definable closed subset of $R^{n}$ of dimension $d$ and $F \in \mathscr{E}^{m}(E)$. By the $\Lambda^{m}$-regular Separation Theorem, decompose $E=M_{1} \cup \cdots \cup M_{s} \cup A$ where

(1) each $M_{i}$ is a $\Lambda^{q}$-pancake of dimension $d$ in a suitable coordinate system;

(2) $A$ is a small, closed, definable subset of $E$;

(3) for all $i \neq j, \operatorname{cl}\left(M_{i}\right), \operatorname{cl}\left(M_{j}\right)$ are $\partial M_{i}$-separated; and

(4) for each $i, \operatorname{cl}\left(M_{i}\right), A$ are $\partial M_{i}$-separated.

By $(*)$, take a definable $C^{m}$-extension $f_{A}$ of $F\left\lceil A\right.$. By replacing $F$ by $F-J^{m}\left(f_{A}\right)\lceil E$,

we may assume that $F$ is flat on $\bigcup_{i=1}^{s} \partial M_{i}$. Now, by separability, Proposition 4.8, and Lemma 5.4, we obtain a $C^{m}$-extension of $F$ which is $C^{q}$ outside $E$.

As usual in the o-minimal context, there is a certain uniformity inherent in the above constructions; this can be exhibited by redoing these construction "uniformly in parameters," or perhaps more elegantly, by using the Compactness Theorem of first-order logic:

Theorem 6.1. Let $\left(F_{a}\right)_{a \in A}$, where $A \subseteq R^{N}$, be a definable family of definable $C^{m}$ Whitney fields $F_{a}$ on a closed definable set $E_{a} \subseteq R^{n}$. Then there is a definable family $\left(f_{a}\right)_{a \in A}$ of definable $C^{m}$-functions $f_{a}: R^{n} \rightarrow R$ such that $f_{a}$ is an extension of $F_{a}$, for each $a \in A$.

Proof. Let $\mathscr{L}$ be the language of $\boldsymbol{R}$, assumed to include a name for each element of $R$, so that every definable set in $R$ is definable by an $\mathscr{L}$-formula. For each $\alpha \in \mathbb{N}^{n}$ with $|\alpha| \leq m$, let $\phi^{\alpha}(x, y, z)$ be a formula in $\mathscr{L}$ where the length of $x, y$, and $z$ are $n, 1$, and $k$, respectively, such that for each $a \in A, \phi^{\alpha}(x, y, a)$ defines the graph of $\left(F_{a}\right)^{\alpha}$. For each formula $\psi(x, y, z)$, let $\chi_{\psi}(z)$ be a formula such that, for each $a \in R^{N}$, $\chi_{\psi}(a)$ holds in $\boldsymbol{R}$ precisely when $\psi(x, y, a)$ defines the graph of a $C^{m}$-extension of $F_{a}$. Next, add $N$ fresh constants $c_{1}, \ldots, c_{N}$ to $\mathscr{L}$ and call the resulting language $\mathscr{L}^{\prime}$. 
For notational convenience, we write $c=\left(c_{1}, \ldots, c_{N}\right)$. By our main theorem, the $\mathscr{L}^{\prime}$-theory

$$
\operatorname{Th}(\boldsymbol{R}) \cup\left\{\neg \chi_{\psi}(c): \psi=\psi(x, y, z) \text { is an } \mathscr{L} \text {-formula }\right\}
$$

is inconsistent. Therefore, by the Compactness Theorem, there are formulas

$$
\psi_{1}(x, y, z), \ldots, \psi_{M}(x, y, z)
$$

such that, for each $a \in A$, one of $\psi_{i}(x, y, a)$ defines the graph of a $C^{m}$-extension of $F_{a}$ in $\boldsymbol{R}$. We can now easily construct a single formula $\psi(x, y, z)$ which works for every $a \in A$, i.e., for each $a \in A, \psi(x, y, a)$ defines the graph of a $C^{m}$-extension of $F_{a}$.

In [9], Pawłucki also shows that his $C^{m}$-extension operator is linear. Unfortunately, in the proof of Lemma 5.1 above, the decomposition depends on each specific $C^{m}$ Whitney field, which results in the loss of linearity. Therefore, we finish this paper with the following open question:

Question. Let $E$ be a definable closed subset of $R^{n}$. Is there an $R$-linear map which assigns to each $F \in \mathscr{E}^{m}(E)$ a definable $C^{m}$-extension of $F$ ?

\section{REFERENCES}

[1] L. van den Dries, Tame Topology and o-minimal Structures, London Mathematical Society Lecture Note Series, vol. 248, Cambridge University Press, Cambridge, 1998.

[2] L. van den Dries, C. Miller, Geometric categories and o-minimal structures, Duke Math. J. 84 (1996), no. 2, 497-540.

[3] A. Fischer, O-minimal $\Lambda^{m}$-regular stratification, Ann. Pure Appl. Logic 147 (2007), no. 1-2, 101-112.

[4] M. Gromov, Entropy, homology and semialgebraic geometry, in: Séminaire Bourbaki, Vol. 1985/86, Astérisque 145-146 (1987), no. 5, 225-240.

[5] M. R. Hestenes, Extension of the range of a differentiable function, Duke Math. J. 8, (1941), 183-192.

[6] K. Kurdyka, W. Pawłucki, Subanalytic version of Whitney's extension theorem, Studia Math. 124 (1997), no. 3, 269-280.

[7] B. Malgrange, Ideals of Differentiable Functions, Oxford University Press, 1966.

[8] W. Pawłucki, A decomposition of a set definable in an o-minimal structure into perfectly situated sets, Ann. Polon. Math. 79 (2002), no. 2, 171-184.

[9] A linear extension operator for Whitney fields on closed o-minimal sets, Ann. Inst. Fourier (Grenoble) $\mathbf{5 8}$ (2008), no. 2, 383-404.

[10] H. Whitney, Analytic extension of differentiable functions defined in closed sets, Trans. Amer. Math. Soc. 36 (1934), 63-89.

[11] Differentiable functions defined in closed sets. I, Trans. Amer. Math. Soc. 36 (1934), 369-389.

[12] $\_$Functions differentiable on the boundaries of regions, Annals of Math. 35 (1934), 482485.

Department of Mathematics, University of California, Los Angeles, los Angeles, CA 90095-1555, U.S.A.

Department of Mathematics, Faculty of Science, Chulalongkorn University, BangKOK 10330, THAILAND

E-mail address: t.athipat@gmail.com 